\title{
Design and Implementation of $n$-Scroll Chaotic Attractors From a General Jerk Circuit
}

\author{
Simin Yu, Jinhu Lü, Member, IEEE, Henry Leung, Member, IEEE, and Guanrong Chen, Fellow, IEEE
}

\begin{abstract}
This paper proposes a novel nonlinear modulating function approach for generating $n$-scroll chaotic attractors based on a general jerk circuit. The systematic nonlinear modulating function methodology developed here can arbitrarily design the swings, widths, slopes, breakpoints, equilibrium points, shapes, and even the general phase portraits of the $n$-scroll chaotic attractors by using the adjustable sawtooth wave, triangular wave, and transconductor wave functions. The dynamic mechanism and chaos generation condition of the general jerk circuit are further investigated by analyzing the system stability. A simple block circuit diagram, including integrator, sawtooth wave and triangular wave generators, buffer, switch linkages, and voltage-current conversion resistors, is designed for the hardware implementations of various 3-12-scroll chaotic attractors via switchings of the switch linkages. This is the first time to experimentally verify a 12-scroll chaotic attractor generated by an analog circuit. In particular, the recursive formulas of system parameters and real physical circuit parameters are rigorously derived for the hardware implementations of the $n$-scroll chaotic attractors. Moreover, the adjustability of the nonlinear modulating function and the rigorous recursive formulas together provide a theoretical principle for the hardware implementations of various chaotic attractors with a large number of scrolls.
\end{abstract}

Index Terms-Jerk circuit, modulating function, sawtooth wave, triangular wave, transconductor wave, $n$-scroll chaotic attractor.

\section{INTRODUCTION}

$\mathbf{O}$ VER the last four decades, chaos has been intensively studied within the science, mathematics, and engineering communities (see, e.g., [1]). Recently, the design and circuit implementation of chaotic oscillators have been a subject of increasing interest due to their applications in various chaos-based technologies and information systems [2]-[38]. Firstly, it provides a powerful tool for further investigating the complicated

Manuscript received July 28, 2004. This work was supported by National Natural Science Foundation of China under Grant 60304017 and Grant 20336040/B06, the Scientific Research Startup Special Foundation on Excellent Ph.D. Thesis and Presidential Award of the Chinese Academy of Sciences, the Natural Science Foundation of Guangdong Province under Grant 32469, the Natural Science Foundation of the Education Department of Guangdong Province under Grant 010042, the Science and Technology Program of Guangzhou City under Grant 2004J1-C0291, and the Hong Kong Research Grants Council under the CERG Grant CityU 1115/03E. This paper was recommended by Associate Editor Y. Nishio.

$\mathrm{S}$. Yu is with the College of Automation, Guangdong University of Technology, Guangzhou 510090, China.

J. Lü is with the Key Laboratory of Systems and Control, the Institute of Systems Science, Academy of Mathematics and Systems Science, Chinese Academy of Sciences, Beijing 100080, China (e-mail: jhlu@iss.ac.cn).

H. Leung is with the Department of Electrical and Computer Engineering, University of Calgary, Calgary, AB T2N 1N4, Canada (e-mail: leungh@ucalgary.ca).

G. Chen is with the Department of Electronic Engineering, City University of Hong Kong, Hong Kong (e-mail: gchen@ee.cityu.edu.hk).

Digital Object Identifier 10.1109/TCSI.2005.851717 dynamical behaviors of chaos oscillators. Secondly, it deepens the understanding of the inherent architectures of chaotic oscillators, which are very useful for systematic electronic design. Moreover, it stimulates the current research on generating various complex multiscroll chaotic attractors by using some simple electronic circuits and devices.

There have been a large number of publications devoted to this research topic of circuits design for generating multiscroll chaotic attractors. Suykens and Vandewalle [2], [3], for instance, proposed a family of $n$-double scroll chaotic attractors, and then Suykens et al. [4] introduced a more complete family of $n$-scroll instead of $n$-double scroll attractors. Suykens and Chua [5] also studied the $n$-double scroll hypercubes in 1-D CNN. A piecewise linear (PWL) implementation of $n$-double scrolls was presented by Arena et al. in [6]. Aziz-Alaoui [7] proposed a method for generating multispiral attractors from both autonomous and nonautonomous differential equations. A family of hyperchaotic $n$-scroll attractors was introduced by Yalcin et al. in [8]. Yalcin et al. [9] also suggested a simple circuit model for creating $n$-scroll chaotic attractors. The main idea of these approaches is the same- to add breakpoints into the piecewise-linear characteristic function of the nonlinear resistor in Chua's circuit [10], [11]. Noticed that hysteresis can also generate chaos [12]. Some hysteresis-based chaotic oscillators were further investigated by Elwakil and Kennedy in [13]. Then, Tang et al. [14], [15] presented a sine-function approach for generating $n$-scroll chaotic attractors, with a systematical circuit realization that can physically produce up to as many as ten scrolls visible on the oscilloscope. A class of circuit-independent chaotic oscillators was constructed by Elwakil and Kennedy in [16]. Ozoguz et al. [17] introduced a nonlinear transconductor approach for creating $n$-scroll attractors. A switching manifold approach for generating chaotic attractors with multiple-merged basins of attraction was proposed by Lü et al. in [18], [19]. Yalcin et al. introduced a family of scroll grid attractors by using a step function approach, including one-directional (1-D) $n$-scroll, two-directional (2-D) $n \times m$-grid scroll, and three-directional (3-D) $n \times m \times l$-grid scroll chaotic attractors [20]. Cafagna and Grassi [21], [22] developed a hyperchaotic coupled Chua's circuit approach by using sine nonlinearity instead of PWL nonlinearity for creating 1-D $n$-scroll, 2-D $n \times m$-grid scroll, and 3-D $n \times m \times l$-grid scroll chaotic attractors. Lü et al. [23]-[27] presented a hysteresis series approach for generating 1-D $n$-scroll, 2-D $n \times m$-grid scroll, and 3-D $n \times m \times l$-grid scroll chaotic attractors, with a rigorously mathematical proof for the chaotic behaviors. More recently, Lü et al. [28], [29] initiated a saturated function series method for creating 1-D $n$-scroll, 2-D $n \times m$-grid scroll, and 
3-D $n \times m \times l$-grid scroll attractors whose chaotic behaviors were verified via a rigorous theoretical approach. Last but not least, Yu et al. [30] introduced a family of hyperchaotic $n$-scroll chaotic attractors in a four order system.

It should be noticed that most of the aforementioned multiscroll chaotic attractors were verified by numerical simulations. However, it is much more difficult to generate $n$-scroll chaotic attractors by physical electronic circuits. In this endeavor, Matsumoto et al. [31] designed a simple circuit to experimentally verify hyperchaotic attractors. Yalcin et al. [32] physically realized a 6-scroll attractor in a generalized Chua's circuit via a rescaling breakpoints approach, and Yalcin et al. [33] experimental confirmed the 3- and 5-scroll chaotic attractors in a generalized Chua's circuit. Elwakil and kennedy [34] proposed a systematic circuit design method for the realization of a class of hysteresis chaotic oscillators, and Elwakil et al. [35] introduced an autonomous system for chaos generation based on a third-order abstract canonical mathematical model with two hardware implementations demonstrated, using commercially available components and CMOS chip. Finally mentioned, Yu et al. [36] constructed a novel circuit to verify $n$-scroll chaotic attractors in a generalized Chua's circuit. Noticed also that it is very difficult to physically realize a nonlinear resistor that has an appropriate characteristic with many segments [15]. In doing so, the main obstacles are: 1 ) the device must have a very wide dynamic range [6], [15], [32]; 2) the slopes of those segments and their breakpoints must be adjustable easily and independently. Yet physical conditions always limit or even prohibit such circuit realizations [15].

It is well known that the Poincaré-Bendixson theorem implies that some necessary conditions for chaos to exist in an autonomous ordinary differential equation (ODE) system are three variables with at least one nonlinearity [37], [38]. Linz and Sprott [39] asked the following basic question: "What are the simplest functional forms of three-dimensional autonomous dynamical systems that still possess chaotic behavior at least for some ranges of the control parameters?" In 1979, Rössler [38] found a toroidal chaotic system of six terms with only one quadratic nonlinearity. In 1994, Sprott [40] found fourteen chaotic systems of six terms with one quadratic nonlinearity and five systems of five terms with two quadratic nonlinearities, via exhaustive computer searching. Zhang and Heidel [41], [42], then, analytically proved that many classes of systems being simpler than Sprott's models cannot be chaotic. Nevertheless, the algebraically simplest chaotic flow has not been identified to date. Further progress in this direction is to consider a special class of three-dimensional dynamical systems-the so-called jerk systems. Their functional forms are described by $\dddot{x}=J(x, \dot{x}, \ddot{x})$, where the first derivative of the position $\dot{x}$ is called velocity, the second $\ddot{x}$ is called acceleration, and the third $\dddot{x}$ is called jerk. In 1996, Gottlieb [41], [42] simplified the basic question as follows: "What is the simplest jerk function that gives chaos?" Sprott [40] answered the question and discovered the algebraically simplest dissipative quadratic form: $\dddot{x}=-\beta \ddot{x}+(\dot{x})^{2}-x$ with $2.017 \leq \beta \leq 2.057$. Eichhorn $e t$ $a l$. [41] further pointed out that the fourteen Sprott's models of six terms with one quadratic nonlinearity as well as the simplest dissipative quadratic flow and Rössler's toroidal model can all be grouped into seven classes of polynomial functions with increasing complexity. The discovery of these simple jerk systems stimulated the present research for a general jerk system [42]: $\dddot{x}+\beta \ddot{x}+\dot{x}=f(x)$, where $f(x)$ is a simple nonlinear function.

Today, it is known that jerk circuits have some practical applications in, for example, broad-band signal generations and secure communications. This is because they are simple circuits that are easy to build, to be re-scaled (to any desired frequencies), and to analyze, predict, and control with very high accuracy [42]. On the other hand, multiscroll chaotic attractors have many practical applications [43], [44], but the aforementioned general jerk system can only generate oneor double-scroll attractors [39]-[42]. Therefore, it is very interesting to ask whether or not the general jerk circuits can be slightly modified so as to generate $n$-scroll chaotic attractors. This paper gives a positive answer to this question. More precisely, this paper proposes a nonlinear modulating function approach for creating $n$-scroll chaotic attractors based on a general jerk circuit. The dynamic mechanism and chaos generation condition of the general jerk circuit are then investigated by analyzing the system stability. In particular, this systematic nonlinear modulating function approach can arbitrarily design the swings, widths, slopes, breakpoints, equilibrium points, shapes, and even some phase portraits of the $n$-scroll chaotic attractors via the adjustable sawtooth wave, triangular wave, and transconductor wave functions. In comparison, most of the reported $n$-scroll attractors can only design different numbers of scrolls and equilibrium points, where many technical parameters such as swings, widths, slopes, shapes, and phase portraits cannot be designed at one's will. In this paper, moreover, the recursive formulas of system parameters and real physical circuit parameters will be rigorously derived for the hardware implementations of the $n$-scroll chaotic attractors. In addition, this paper reports for the first time an experimental verification of a 12-scroll chaotic attractor.

The rest of the paper is organized as follows. In Section II, a general jerk circuit is introduced and some conditions for chaos generation are derived. The proposed design approach is then discussed in Section III, for generating $n$-scroll chaotic attractors via the general jerk circuit. In Section IV, circuit implementations of the chaotic attractors with a large number of scrolls are further investigated, and the recursive formulas of system parameters and real physical circuit parameters are also rigorously derived. Conclusions are finally drawn in Section V.

\section{General Jerk Circuits}

In this section, a general jerk circuit is introduced and some conditions for chaos generation are derived.

\section{A. General Jerk Circuit}

The general jerk circuit is described by

$$
\dddot{x}+\beta \ddot{x}+\gamma \dot{x}=f(x)
$$

where $\beta, \gamma$ are real parameters, $f(x)$ is a nonlinear function, $\dot{x}=((d x) /(d \tau))$ is the velocity, $\ddot{x}=\left(\left(d^{2} x\right) /\left(d \tau^{2}\right)\right)$ is the acceleration, $\ddot{x}=\left(\left(d^{3} x\right) /\left(d \tau^{3}\right)\right)$ is the jerk (or, the rate of change of the acceleration by mechanical means), $\tau=\left(t /\left(R_{0} C_{0}\right)\right)$, in 


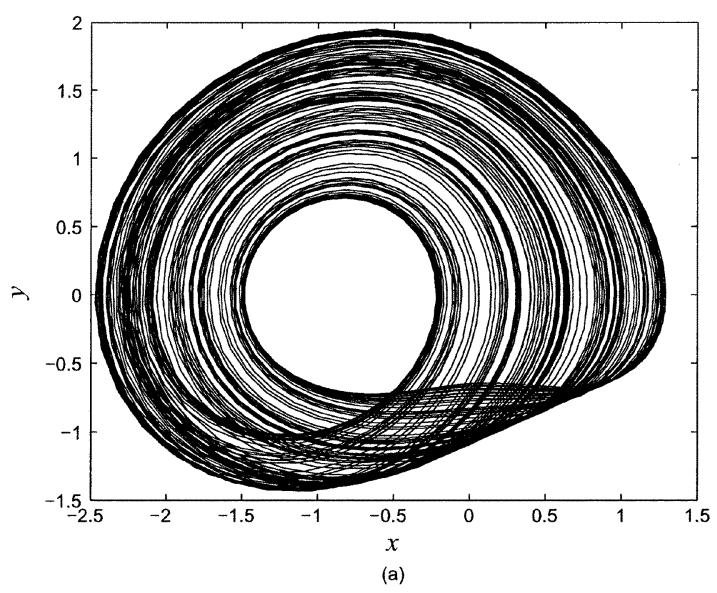

Fig. 1. Chaotic attractors of jerk system (1). (a) Single-scroll. (b) Double-scroll.

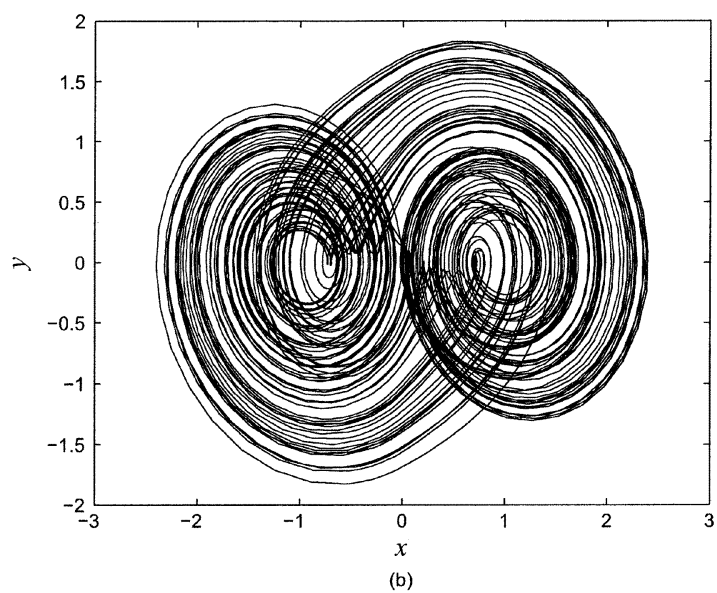

The following is a result for the stability of equilibrium point $\left(x_{0}, 0,0\right)$ of the jerk system (1).

Lemma 1: Assume that $f(x)$ is differentiable at the equilibrium point $\left(x_{0}, 0,0\right)$. A necessary and sufficient condition for the stability of the equilibrium point $\left(x_{0}, 0,0\right)$ is that $\beta>0$ and $-\beta \gamma<f^{\prime}<0$, where $f^{\prime}=\left.((d f) /(d x))\right|_{x=x_{0}}$.

Proof: The characteristic equation of the jerk system (1) is

$$
\lambda^{3}+\beta \lambda^{2}+\gamma \lambda-f^{\prime}=0
$$

where $f^{\prime}=\left.((d f) /(d x))\right|_{x=x_{0}}$. From the Routh-Hurwitz Criterion, the real parts of the roots $\lambda$ are negative if and only if the following conditions hold:

$$
\beta>0, \quad-f^{\prime}>0, \quad \beta \gamma+f^{\prime}>0 .
$$

That is, the equilibrium point $\left(x_{0}, 0,0\right)$ is stable if and only if $\beta>0$ and $-\beta \gamma<f^{\prime}<0$. Thus, the proof is completed.

It is easy to see that the jerk system (1) undergoes a Hopf bifurcation at $f^{\prime}=-\beta \gamma$, where $\lambda= \pm i$ for $\gamma=1$. According to Lemma 1, one should design some equilibrium points to satisfy $f^{\prime}>0$ or $f^{\prime}<-\beta \gamma$ in order to generate chaos in system (1). That is, one should design the nonlinearity with either a positive slope at its equilibrium point or a suitable negative slope that implies a negative resistance in the corresponding circuit [41]. The jerk system (1) with $f^{\prime}>0$ apparently requires at least two equilibrium points for chaos generation; however, system (1) with $f^{\prime}<-\beta$ only need one [41].

Jerk circuits are easy to build and to be re-scaled over a wide range of frequencies. Moreover, jerk circuits have a variety of dynamical behaviors and are similar in spirit to Chua's circuit [10], [11]. However, Chua's circuit has a very complicated analytical form in terms of $\ddot{x}$, with more than four terms including step functions, delta functions, and their products with derivatives of $x$. Therefore, such a Chua's circuit is difficult to construct, to re-scale, and to analyze, due also to the needed inductor with its frequency-dependent resistive losses [42].

Denote $p=\gamma-(1 / 3) \beta^{2}, q=(2 / 27) \beta^{3}-(1 / 3) \beta \gamma-$ $f^{\prime}$, and $\Delta=-\left(\left(\beta^{3} f^{\prime}\right) / 27\right)-\left(\left(\beta^{2} \gamma^{2}\right) / 108\right)+\left(\left(\beta \gamma f^{\prime}\right) / 6\right)+$ $\left(\left(\gamma^{3}\right) / 27\right)+\left(\left(f^{\prime 2}\right) / 4\right)$. Then, solving (3) gives

$$
\lambda_{1}=-\frac{\beta}{3}+\sqrt[3]{-\frac{q}{2}+\sqrt{\Delta}}+\sqrt[3]{-\frac{q}{2}-\sqrt{\Delta}}
$$




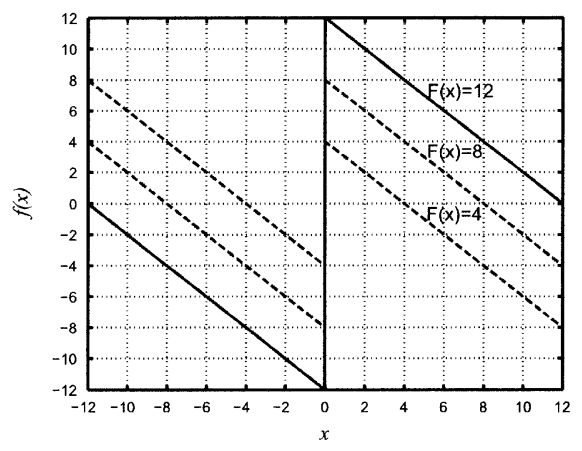

(a)

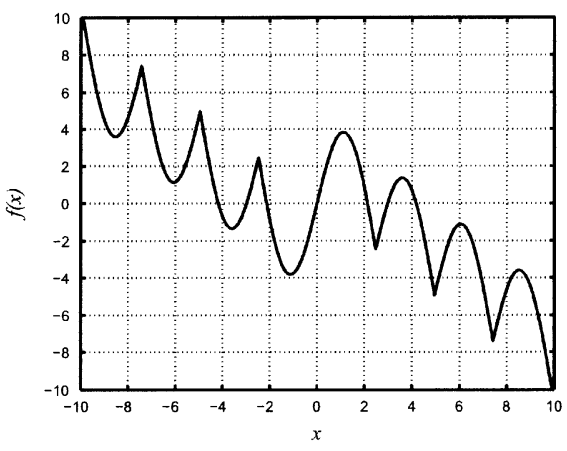

(b)

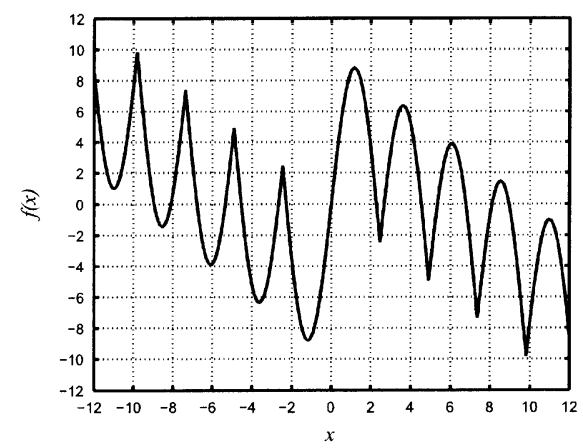

(c)

Fig. 2. Constructing graph of $f(x)=|F(x)| \operatorname{sgn}(x)-x$. (a) $F(x)=4,8,12$. (b) $F(x)=5 \sin (1.27 x)$; (c) $F(x)=10 \sin (1.28 x)$.

and

$$
\begin{aligned}
\lambda_{2,3} & =-\frac{\beta}{3}-\frac{1}{2}\left(\sqrt[3]{-\frac{q}{2}+\sqrt{\Delta}}+\sqrt[3]{-\frac{q}{2}-\sqrt{\Delta}}\right) \\
& \pm \frac{\sqrt{3}}{2} i\left(\sqrt[3]{-\frac{q}{2}+\sqrt{\Delta}}-\sqrt[3]{-\frac{q}{2}-\sqrt{\Delta}}\right) \\
& \equiv \alpha \pm \xi i .
\end{aligned}
$$

Obviously, for $\beta=0.6, \gamma=1, p=0.88, q=-0.184-f^{\prime}$, and $\Delta=0.25 f^{\prime 2}+0.092 f^{\prime}+(7 / 1500)$. If $\Delta>0$, then $f^{\prime}>((-0.552+2 \sqrt{0.034176}) / 3) \approx-0.0608$ and $f^{\prime}<$ $((-0.552-2 \sqrt{0.034176}) / 3) \approx-0.3072$. Numerical calculation shows that the jerk system (1) may generate chaos under the conditions that $\Delta>0, \lambda_{1}<0$, and $\alpha>0$. That is, there exists a saddle point of index 2 in system (1), [27]-[29]. Indeed, the saddle points of index 2 play a key role in the chaos generation in system (1).

\section{Design OF $n$-SCROLl ChaOtiC AtTRACTORS}

In this section, a systematic design approach is presented for generating $n$-scroll chaotic attractors in the general jerk system (1).

\section{A. Designing Scroll Nesting Chaotic Attractors via Modulating Function}

In this subsection, a swing modulating function of double sawtooth wave is constructed to generate multiscroll chaotic attractors in the jerk system (1). The function is described by

$$
f(x)=|F(x)| \operatorname{sgn}(x)-x
$$

where $F(x)$ is the swing modulating function, which controls the swings of scrolls and equilibrium points of system (1). Fig. 2 shows the constructing graph of $f(x)$. In more detail, Fig. 2(a) shows the constant case for $F(x)=4,8,12$; Fig. 2(b) and (c) shows the sine function cases for $F(x)=5 \sin (1.27 x)$ and $F(x)=10 \sin (1.28 x)$, respectively. When the modulating function increases, system (1) with (6) creates a large-scale double-scroll attractor; when the modulating function decreases, system (1) with (6) generates a smaller double-scroll attractor. Especially, when the modulating function varies with variable $x$, system (1) with (6) produces various nesting double-scrolls to form a complex multiscroll attractor.

It is noticed that the modulating function $F(\cdot)$ may be a autonomous function, or a nonautonomous function produced by external signals. Of course, $F(\cdot)$ can be a constant in the special case. To generate chaos in system (1) with (6), the modulating function $F(\cdot)$ has to satisfy some conditions. In the following, assume that

$$
F(x)=A \sin (a x)
$$

where $A, a>0$ are parameters.

If $(1 / A) \geq a, f(x)$ has a unique zero, $x_{0}=0$; if $((2 a) /(3 \pi))<(1 / A)<a, f(x)$ has three zeros, $x_{0}, x_{ \pm 1}$; if $(1 / A)=((2 a) /((2 k+1) \pi))$ for $k \in N, f(x)$ has $4 k+1$ zeros, $x_{0}, x_{ \pm i}(i=1, \ldots, \pm 2 k)$; if $((2 a) /((2 k+3) \pi))<$ $(1 / A)<((2 a) /((2 k+1) \pi))$ for $k \in N, f(x)$ has $4 k+3$ zeros, $x_{0}, x_{ \pm i}(i=1, \ldots, \pm(2 k+1))$. Obviously, $f^{\prime}=A a \cos (a x)-1$ for $((2 k \pi) / a) \leq x \leq(((2 k+1) \pi) / a)(k=0,1, \ldots)$ and $(((2 k-1) \pi) / a) \leq x \leq((2 k \pi) / a)(k=0,-1, \ldots)$, and $f^{\prime}=-A a \cos (a x)-1$ for $(((2 k-1) \pi) / a) \leq$ $x \leq((2 k \pi) / a)(k=1,2, \ldots)$ and $(2 k \pi / a) \leq x \leq$ $((2 k+1) \pi / a)(k=-1,-2, \ldots)$.

It is easy to verify the stabilities of the equilibrium points $\left(x_{i}, 0,0\right)$ of the jerk system (1) with (6) and (7), by using Lemma $1,(4)$ and (5) together.

Assume that $A=5, a=1.27$. Then, system (1) with (6) and (7) has a scroll-nesting 4-scroll attractor, as shown in Fig. 3(a) and (b). Similarly, assume that $A=10, a=1.28$. Then, system (1) with (6) and (7) has a scroll-nesting 8-scroll attractor, as shown in Fig. 3(c) and (d).

\section{B. Designing Multiscroll Chaotic Attractors via Adjustable Sawtooth Wave}

In this subsection, an adjustable sawtooth wave is constructed for generating various multiscroll chaotic attractors.

To create the chaotic attractor with an even number of scrolls, the adjustable sawtooth wave is described by

$$
\begin{aligned}
f_{1}(x) & =A_{0} \operatorname{sgn}(x)+\sum_{i=1}^{M}\left[\frac{A_{i-1}+A_{i}}{2} \operatorname{sgn}\left(x-\frac{2}{B} \sum_{j=0}^{i-1} A_{j}\right)\right] \\
& +\sum_{i=1}^{M}\left[\frac{A_{i-1}+A_{i}}{2} \operatorname{sgn}\left(x+\frac{2}{B} \sum_{j=0}^{i-1} A_{j}\right)\right]-B x
\end{aligned}
$$

where all parameters $A_{i}>0(i=0,1,2, \ldots)$ and $B \in[0.7,1.2]$, which can generate $2 M+2(M=1,2,3, \ldots)$ scrolls in a attractor. 


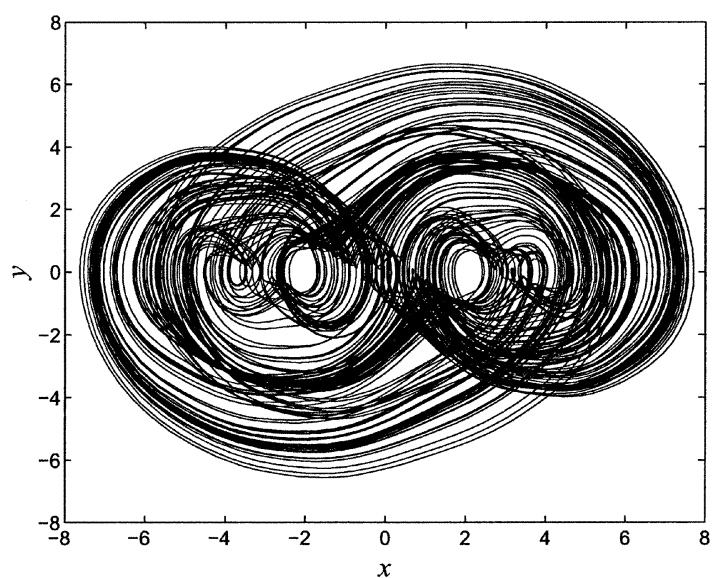

(a)

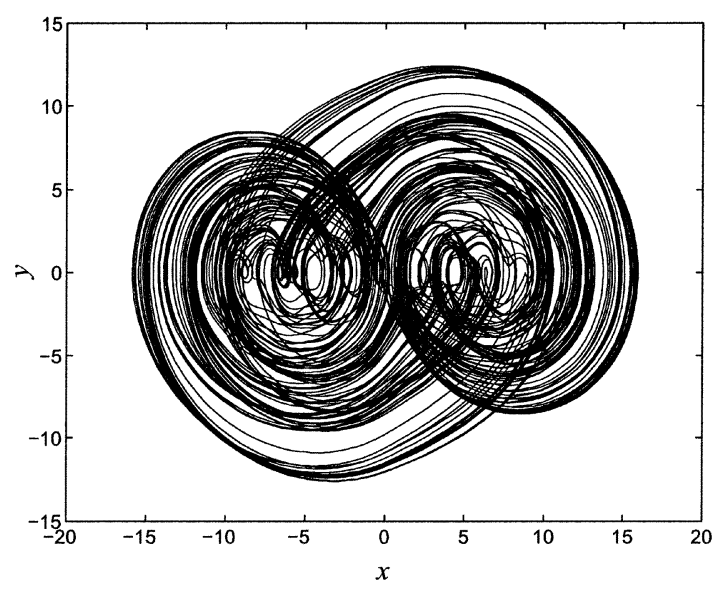

(c)

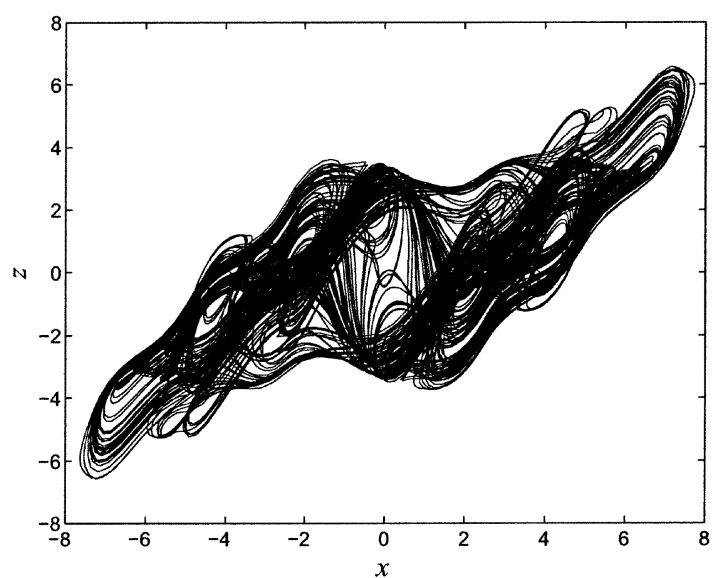

(b)

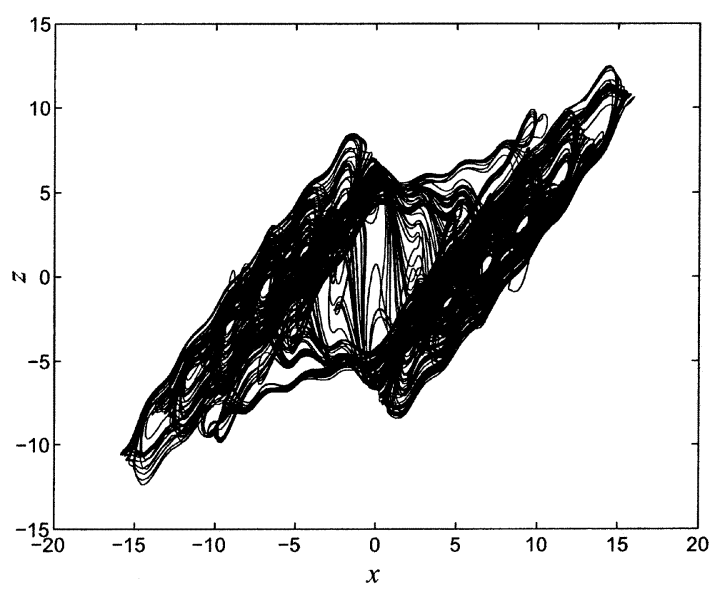

(d)

Fig. 3. Scroll nesting chaotic attractor. (a) and (b) 4 -scroll $(A=5, \alpha=1.27)$. (c) and (d) 8 -scroll $(A=10, \alpha=1.28)$.

Similarly, to generate the chaotic attractor with an odd number of scrolls, the adjustable sawtooth wave is described by

$$
\begin{aligned}
f_{2}(x)= & \sum_{i=1}^{M}\left\{\frac{A_{i-1}+A_{i}}{2} \operatorname{sgn}\left[x-\frac{1}{B}\left(2 \sum_{j=0}^{i-1} A_{j}-A_{0}\right)\right]\right\} \\
& +\sum_{i=1}^{M}\left\{\frac{A_{i-1}+A_{i}}{2} \operatorname{sgn}[x\right. \\
& \left.\left.+\frac{1}{B}\left(2 \sum_{j=0}^{i-1} A_{j}-A_{0}\right)\right]\right\}-B x
\end{aligned}
$$

where all parameters $A_{i}>0(i=0,1,2, \ldots)$ and $B \in[0.7,1.2]$, which can create $2 M+1(M=1,2,3, \ldots)$ scrolls in the attractor.

Note that all characteristic quantities of the multiscroll chaotic attractor, including the swing, width, slope, and equilibrium points, can be determined beforehand by the system parameters $A_{i}(i=0,1, \ldots, M)$ and $B$. By adjusting these parameters, one can arbitrarily design the swing, width, slope, and equilibrium points, to generate various multiscroll attractors. For example, when $A_{0}=A_{1}=\cdots=A_{M}$, systems (8) and (9) produce multiscroll attractors with the same swing and
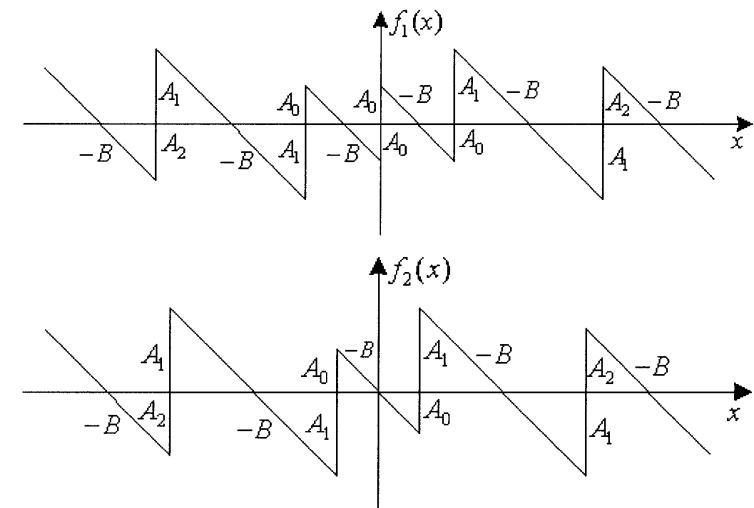

Fig. 4. Adjustable sawtooth wave $f_{1}(x)$ and $f_{2}(x)$.

width via the sawtooth wave with the same swing and width, which is a special case of the general adjustable sawtooth wave. Fig. 4 shows the adjustable sawtooth waves $f_{1}(x)$ in (8) and $f_{2}(x)$ in (9).

Here, assume that: (i) $f_{1}(x)$ and $f_{2}(x)$ are odd functions; (ii) the zeros of $f_{1}(x)$ and $f_{2}(x)$ lie in the centers of two neighboring breakpoints. Then, one can rigorously deduce a set of recursive formulas on the parameters of sawtooth wave. Due to the symmetry of the odd functions $f_{1}(x)$ and $f_{2}(x)$, one only needs to 
consider the case $x \geq 0$ for the sawtooth wave. One can derive the recursive formulas as follows.

1) The slopes of the sawtooth waves $f_{1}(x)$ and $f_{2}(x)$ are $-B$, satisfying $B \in[0.7,1.2]$.

2) Denote the swings of the scrolls of the sawtooth waves $f_{1}(x)$ and $f_{2}(x)$ by $E_{i}(i=0,1, \ldots, M)$. Then, the recursive formulas of $E_{i}$ are

$$
E_{0}=2 A_{0}, \quad E_{i}=A_{i-1}+A_{i}
$$

where $i=1, \ldots, M$.

3) Denote the widths between two neighboring scrolls of the sawtooth waves $f_{1}(x)$ and $f_{2}(x)$ by $W_{i}(i=$ $0,1, \ldots, M-1)$. Then, the recursive formulas of $W_{i}$ (except the outside edge scroll) are

$$
W_{i}=\frac{2 A_{i}}{B}
$$

where $i=0,1, \ldots, M-1$.

4) Denote the breakpoints of the sawtooth waves $f_{1}(x)$ and $f_{2}(x)$ by $S_{i}(i=0,1, \ldots, M)$. Then, the recursive formulas of $S_{i}$ are

$$
\left\{\begin{array}{l}
S_{i, f_{1}}=\frac{1}{B} \sum_{j=0}^{i-1} 2 A_{j} \\
S_{i, f_{2}}=\frac{1}{B}\left(\sum_{j=0}^{i-1} 2 A_{j}-A_{0}\right)
\end{array}\right.
$$

where $i=1,2, \ldots, M$.

5) Denote the zeros of the sawtooth waves $f_{1}(x)$ and $f_{2}(x)$ by $P_{i}(i=0,1, \ldots, M)$. Then, the recursive formulas of $P_{i}$ are

$$
\left\{\begin{array}{l}
P_{i, f_{1}}=\frac{1}{B}\left(\sum_{j=0}^{i-1} 2 A_{j}-A_{i-1}\right) \\
P_{i, f_{2}}=\frac{1}{B}\left(\sum_{j=0}^{i-1} 2 A_{j}-A_{i-1}-A_{0}\right)
\end{array}\right.
$$

where $i=1, \ldots, M$.

Moreover, the stabilities of the equilibrium points $\left(P_{i, f_{j}}, 0,0\right)$ for $i=1, \ldots, M$ and $j=1$ (or $j=2$ ) of the jerk system (1) with (8) [or (9)] can be confirmed by Lemma 1, (4) and (5).

In the following, all the parameters of the chaotic attractor with even number of scrolls are calculated by using the recursive formulas (10)-(13). Let $M=5$, so that $2 M+2=12$. It is further investigated four kinds of 12-scroll chaotic attractors with different sizes. That is, Type I: multiscroll attractors, with the sizes of the scrolls gradually increasing from the center to both sides; Type II: multiscroll attractors, with the sizes of the scrolls gradually decreasing from the center to both sides; Type III: multiscroll attractors, with the scrolls alternating between small and large scrolls; Type IV: multiscroll attractors, with all scrolls being same in size. Tables $\mathrm{I}-\mathrm{V}$ show the detailed parameters values for $A_{i}(0 \leq i \leq 5)$, slope $-B$, swings $E_{i}(0 \leq i \leq 5)$, widths $W_{i}(0 \leq i \leq 4)$, breakpoints $S_{i}(0 \leq i \leq 5)$, and zeros $P_{i}(0 \leq i \leq 5)$ of the 12 -scroll attractor.
TABLE I

PARAMETERS $A_{i}(0 \leq i \leq 5)$ AND $B$ OF 11 - AND 12-SCROLl ATTRACTORS

\begin{tabular}{c|c|c|c|c|c|c|c}
\hline Type & $A_{0}$ & $A_{1}$ & $A_{2}$ & $A_{3}$ & $A_{4}$ & $A_{5}$ & $B$ \\
\hline I & 0.30 & 0.34 & 0.38 & 0.42 & 0.46 & 0.50 & 1.00 \\
\hline II & 0.50 & 0.46 & 0.42 & 0.38 & 0.34 & 0.30 & 1.00 \\
\hline III & 0.35 & 0.25 & 0.35 & 0.25 & 0.35 & 0.25 & 1.00 \\
\hline IV & 0.25 & 0.25 & 0.25 & 0.25 & 0.25 & 0.25 & 1.00 \\
\hline
\end{tabular}

TABLE II

SWINGS $E_{i}(0 \leq i \leq 5)$ OF 12-SCROLl ATTRACTOR

\begin{tabular}{c|c|c|c|c|c|c}
\hline Type & $E_{0}$ & $E_{1}$ & $E_{2}$ & $E_{3}$ & $E_{4}$ & $E_{5}$ \\
\hline I & 0.60 & 0.64 & 0.72 & 0.80 & 0.88 & 0.96 \\
\hline II & 1.00 & 0.96 & 0.88 & 0.80 & 0.72 & 0.64 \\
\hline III & 0.70 & 0.60 & 0.60 & 0.60 & 0.60 & 0.60 \\
\hline IV & 0.50 & 0.50 & 0.50 & 0.50 & 0.50 & 0.50 \\
\hline
\end{tabular}

TABLE III

WIDTHS $W_{i}(0 \leq i \leq 4)$ OF 11 - AND 12-SCROLL ATTRACTORS

\begin{tabular}{c|c|c|c|c|c}
\hline Type & $W_{0}$ & $W_{1}$ & $W_{2}$ & $W_{3}$ & $W_{4}$ \\
\hline I & 0.60 & 0.68 & 0.76 & 0.84 & 0.92 \\
\hline II & 1.00 & 0.92 & 0.84 & 0.76 & 0.68 \\
\hline III & 0.70 & 0.50 & 0.70 & 0.50 & 0.70 \\
\hline IV & 0.50 & 0.50 & 0.50 & 0.50 & 0.50 \\
\hline
\end{tabular}

TABLE IV

BREAKPOINTS $S_{i}(0 \leq i \leq 5)$ OF 12-SCROLL ATTRACTOR

\begin{tabular}{c|c|c|c|c|c|c}
\hline Type & $S_{0}$ & $S_{1}$ & $S_{2}$ & $S_{3}$ & $S_{4}$ & $S_{5}$ \\
\hline I & 0.00 & 0.60 & 1.28 & 2.04 & 2.88 & 3.80 \\
\hline II & 0.00 & 1.00 & 1.92 & 2.76 & 3.52 & 4.20 \\
\hline III & 0.00 & 0.70 & 1.20 & 1.90 & 2.40 & 3.10 \\
\hline IV & 0.00 & 0.50 & 1.00 & 1.50 & 2.00 & 2.50 \\
\hline
\end{tabular}

TABLE V

Zeros $P_{i}(0 \leq i \leq 5)$ OF 12-SCROLl ATtRACTOR

\begin{tabular}{c|c|c|c|c|c|c}
\hline Type & $P_{0}$ & $P_{1}$ & $P_{2}$ & $P_{3}$ & $P_{4}$ & $P_{5}$ \\
\hline I & 0.30 & 0.94 & 1.66 & 2.46 & 3.34 & 4.30 \\
\hline II & 0.50 & 1.46 & 2.34 & 3.14 & 3.86 & 4.50 \\
\hline III & 0.35 & 0.95 & 1.55 & 2.15 & 2.75 & 3.35 \\
\hline IV & 0.25 & 0.75 & 1.25 & 1.75 & 2.25 & 2.75 \\
\hline
\end{tabular}

TABLE VI

SWINGS $E_{i}(1 \leq i \leq 5)$ OF 11 -SCROLL ATTRACTOR

\begin{tabular}{c|c|c|c|c|c}
\hline Type & $E_{1}$ & $E_{2}$ & $E_{3}$ & $E_{4}$ & $E_{5}$ \\
\hline I & 0.64 & 0.72 & 0.80 & 0.88 & 0.96 \\
\hline II & 0.96 & 0.88 & 0.80 & 0.72 & 0.64 \\
\hline III & 0.60 & 0.60 & 0.60 & 0.60 & 0.60 \\
\hline IV & 0.50 & 0.50 & 0.50 & 0.50 & 0.50 \\
\hline
\end{tabular}

Similarly, one can calculate all the parameters of the chaotic attractor with odd number of scrolls by using the recursive formulas (10)-(13). Let $M=5$, so that $2 M+1=11$. It is to further investigate four kinds of 11-scroll chaotic attractors with different sizes, including Types I, II, III, IV specified above. Tables I, III, and VI-VIII show the detailed parameters values for $A_{i}(0 \leq i \leq 5), B$, swings $E_{i}(0 \leq i \leq 5)$, widths $W_{i}(0 \leq$ $i \leq 4)$, breakpoints $S_{i}(0 \leq i \leq 5)$, and zeros $P_{i}(0 \leq i \leq 5)$ of the 11-scroll attractor, respectively. 


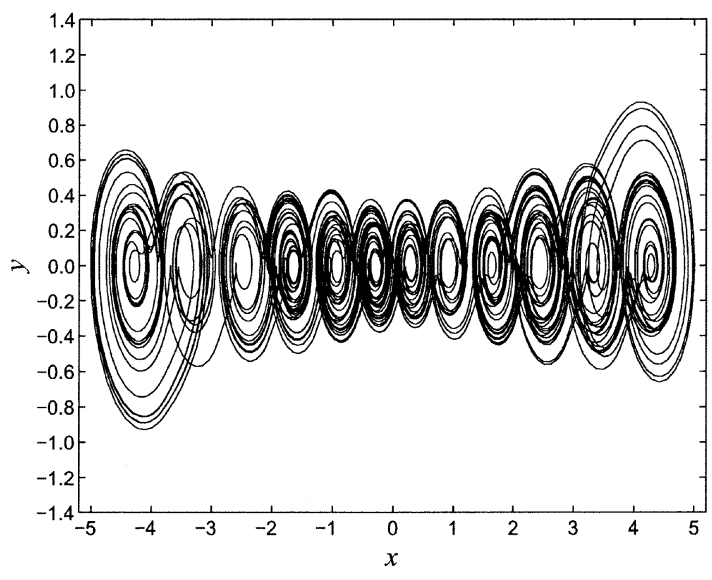

(a)

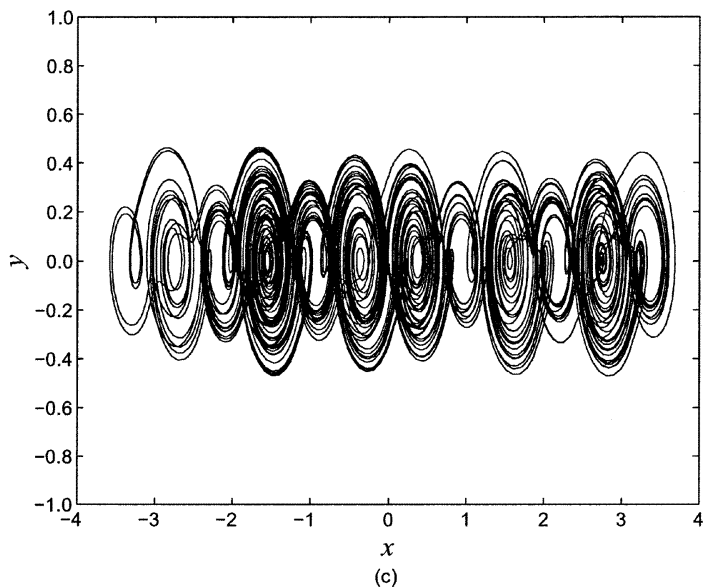

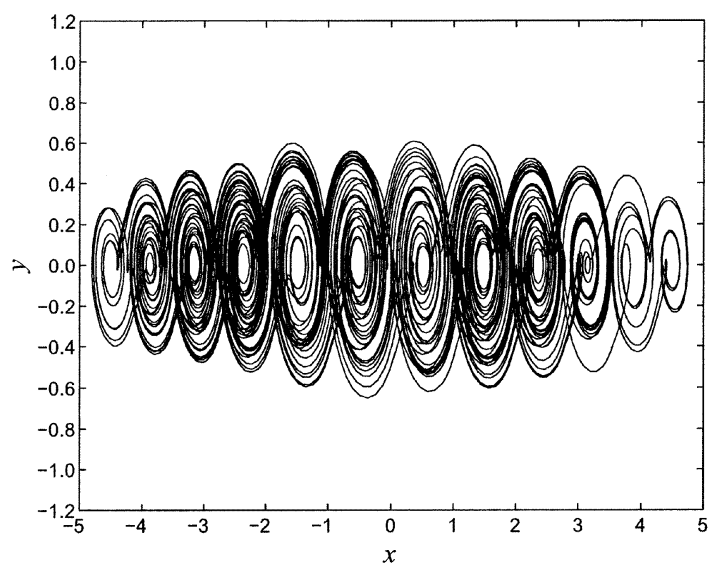

(b)

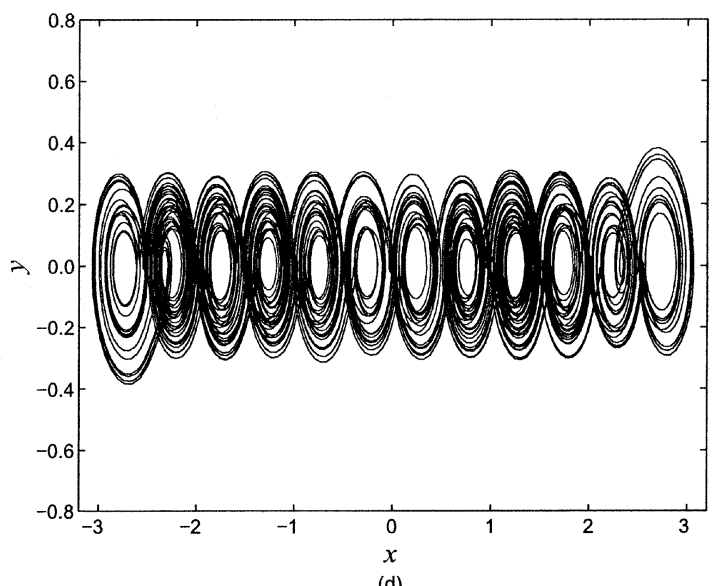

(d)

Fig. 5. Numerical simulations of a 12-scroll attractor. (a) Type I. (b) Type II. (c) Type III. (d) type IV.

TABLE VII

BREAKPOINTS $S_{i}(1 \leq i \leq 5)$ OF 11-SCROLL ATTRACTOR

\begin{tabular}{c|c|c|c|c|c}
\hline Type & $S_{1}$ & $S_{2}$ & $S_{3}$ & $S_{4}$ & $S_{5}$ \\
\hline I & 0.30 & 0.98 & 1.74 & 2.58 & 3.50 \\
\hline II & 0.50 & 1.42 & 2.26 & 3.02 & 3.70 \\
\hline III & 0.35 & 0.85 & 1.55 & 2.05 & 2.75 \\
\hline IV & 0.25 & 0.75 & 1.25 & 1.75 & 2.25 \\
\hline
\end{tabular}

TABLE VIII

ZEROS $P_{i}(0 \leq i \leq 5)$ OF 11 -SCROLl ATtRACTOR.

\begin{tabular}{c|c|c|c|c|c|c}
\hline Type & $P_{0}$ & $P_{1}$ & $P_{2}$ & $P_{3}$ & $P_{4}$ & $P_{5}$ \\
\hline I & 0.00 & 0.64 & 1.36 & 2.16 & 3.04 & 4.00 \\
\hline II & 0.00 & 0.96 & 1.84 & 2.64 & 3.36 & 4.00 \\
\hline III & 0.00 & 0.60 & 1.20 & 1.80 & 2.40 & 3.00 \\
\hline IV & 0.00 & 0.50 & 1.00 & 1.50 & 2.00 & 2.50 \\
\hline
\end{tabular}

According to (1), (8), and Table I, one can get four kinds of 12-scroll chaotic attractors, as shown in Fig. 5. From (1), (9), and Table I, one can get four kinds of 11-scroll chaotic attractor, as shown in Fig. 6.

\section{Designing Multiscroll Chaotic Attractors via Adjustable Triangular Wave}

In this subsection, an adjustable triangular wave is constructed, to create various multiscroll chaotic attractors.

In most chaotic circuits, such as Chua's circuit, four-dimensional MCK chaotic circuit, and some Sprott's chaotic jerk circuits, their PWL functions have constant breakpoints and slopes. In the following, a PWL function with varying breakpoints and slopes is constructed, to generate single-scroll and double-scroll attractors in the jerk system (1). The function is described by

$$
\begin{aligned}
f(x) & =\frac{A}{2 \alpha}(|x+\alpha|-|x-\alpha|)-B x \\
& = \begin{cases}-B x-A, & x<-\alpha \\
\frac{A-\alpha B}{\alpha} x, & -\alpha \leq x \leq \alpha \\
-B x+A, & x>\alpha\end{cases}
\end{aligned}
$$

where parameters $A>0, B \in[0.8,1.2]$, and $\alpha \in(0,(A / B)]$ represents the varying breakpoints.

Fig. 7 shows the evolving graph of $f(x)$ with parameter $\alpha$, where the slopes of two side radials are $k=-B$, and the slope of the middle segment is $k=((A-\alpha B) / \alpha)$.

In system (1) with (14), assume that $A=B=1$. Then, $\alpha \in(0,1]$. Fig. 8(a) shows the bifurcation graph of the breaking parameter $\alpha$ of system (1) with (14). Fig. 8(b) displays the Lyapunov exponent spectrum of the breaking parameter $\alpha$ of system (1) with (14). Fig. 8(c) shows the maximum Lyapunov exponent spectrum of the breaking parameter $\alpha$ of system (1) with (14). Fig. 8(d) displays the power spectrum of the breaking parameter $\alpha$ of double-scroll attractor. It is clear from Fig. 8(a) that system (1) gradually evolves into a chaotic region through a typical doubling-period bifurcation route, where the black areas are chaotic areas and the white areas in the black areas are periodic 


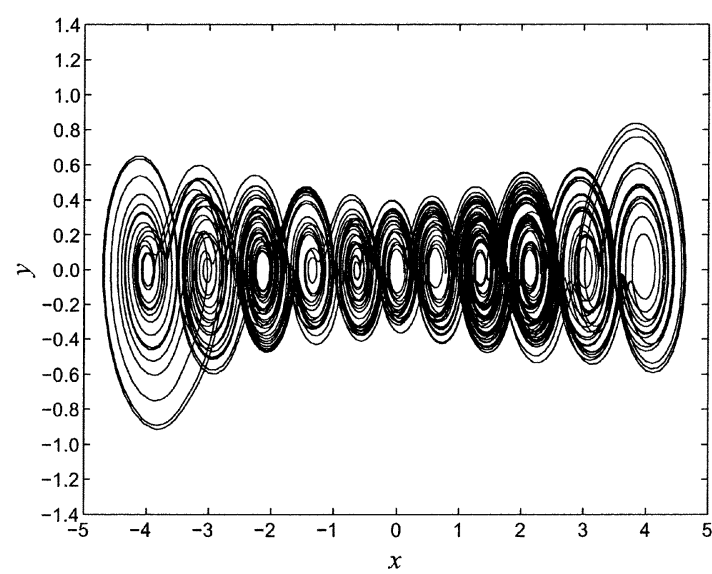

(a)

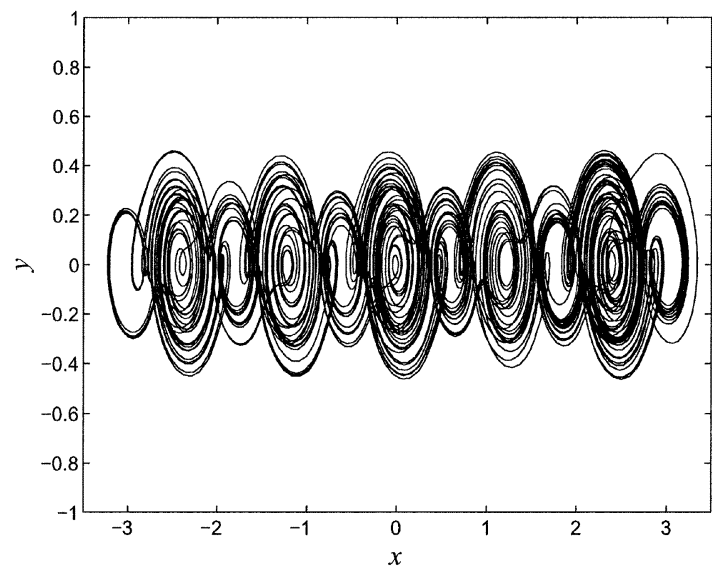

(c)

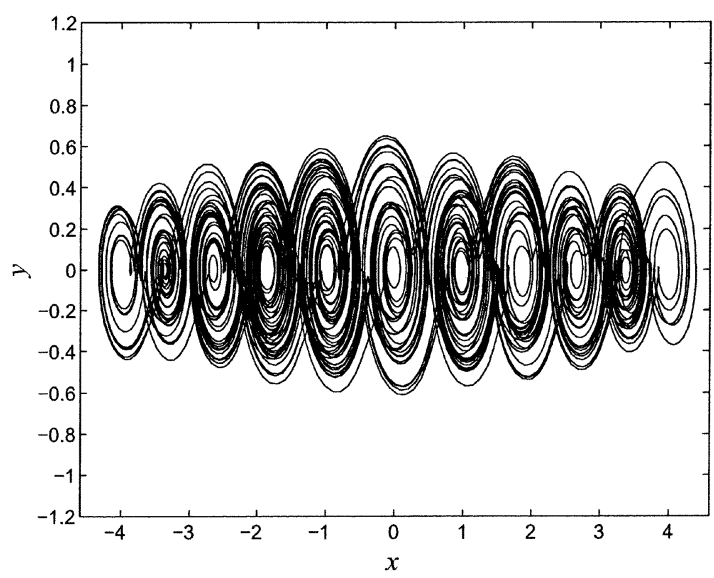

(b)

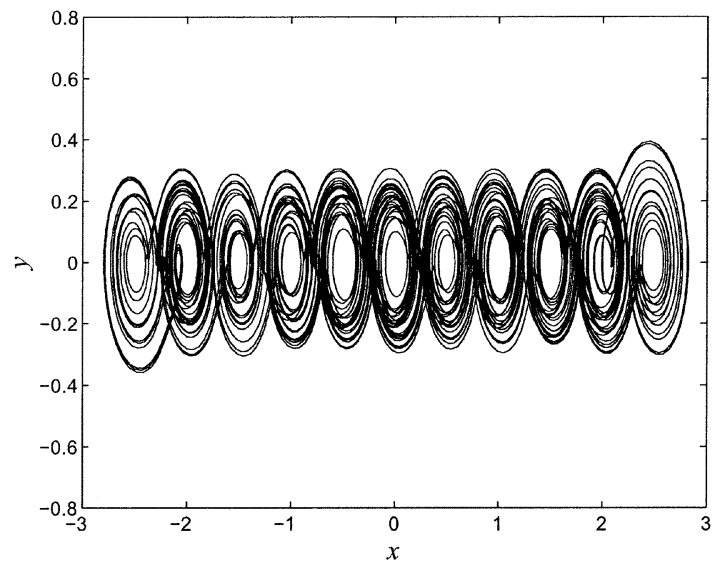

(d)

Fig. 6. Numerical simulations of a 11-scroll attractor. (a) Type I. (b) Type II. (c) Type III. (d) Type IV.

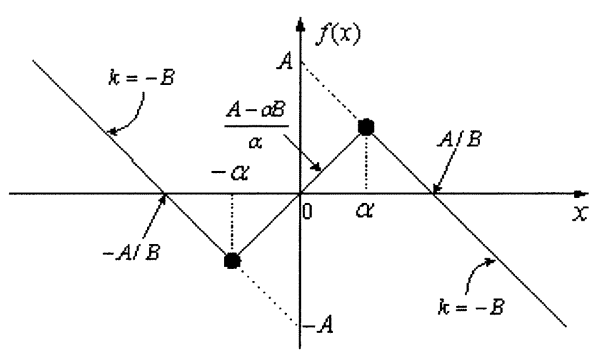

Fig. 7. PWL odd function $f(x)$ with varying breaking parameter $\alpha$.

windows. Further numerical investigations reveal that system (1) with (14) can generate both single- and double-scroll attractors in the chaotic region. When $0.465<\alpha<0.483$ and $0.50<\alpha<0.52$, there exists a single-scroll attractor, as shown in Fig. 8(e); when $0<\alpha<0.3$, there exists a double-scroll attractor, as shown in Fig. 8(f). It is very interesting to see that the single-scroll and double-scroll coexist in the chaotic region of system (1) with (14).

Based on (14), to generate the chaotic attractor with an even number of scrolls, the adjustable triangular wave is constructed as

$$
\begin{aligned}
f_{1}(x)=\sum_{n=-M}^{M} \frac{A}{2 \alpha_{n}}[\mid & \left(x-\frac{2 A n}{B}\right)+\alpha_{n} \mid \\
& \left.-\left|\left(x-\frac{2 A n}{B}\right)-\alpha_{n}\right|\right]-B x
\end{aligned}
$$

where parameters $A>0,0.8 \leq B \leq 1.2, \alpha_{n} \in(0,((3 A) /$ $10 B)](n=0, \pm 1, \ldots, \pm M), M=1,2, \ldots$, which can create $2 M+2$ scrolls in the chaotic attractor.

Similarly, to create a chaotic attractor with an odd number of scrolls, the adjustable triangular wave is constructed as

$$
\begin{aligned}
f_{2}(x)=\sum_{\substack{n=-M \\
n \neq 0}}^{M} \frac{A}{2 \alpha_{n}}\left[\left|\left(x-\frac{A}{B}\left(2 n-\frac{|n|}{n}\right)\right)+\alpha_{n}\right|\right. \\
\left.-\left|\left(x-\frac{A}{B}\left(2 n-\frac{|n|}{n}\right)\right)-\alpha_{n}\right|\right]-B x
\end{aligned}
$$

where parameters $A>0,0.8 \leq B \leq 1.2, \alpha_{n} \in$ $(0,((3 A / 10 B))](n= \pm 1, \pm 2, \ldots, \pm M), M=1,2, \ldots$, which can create $2 M+1$ scrolls in the chaotic attractor.

It is noticed that the characteristic quantities of the multiscroll attractor, such as the swings, widths, and slopes, can be determined by parameters $A, B, \alpha_{n}$. Moreover, the negative slopes of the above triangular waves are $k_{-}=-B$; and the positive slopes of the above triangular waves are $k_{+}=\left(\left(A-\alpha_{n} B\right) / \alpha_{n}\right)$. Especially, when $\alpha_{n} \rightarrow 0$, the triangular wave moves to the sawtooth wave. Furthermore, the following are true.

1) If $f_{1}(x)$ and $f_{2}(x)$ are odd functions, then $\alpha_{i}=\alpha_{-i}$ for $i=1,2, \ldots, M$.

2) Parameters $\alpha_{n}$ can adjust the swings and widths of the scrolls of the multiscroll attractor. If all $\alpha_{n}$ are same, all 


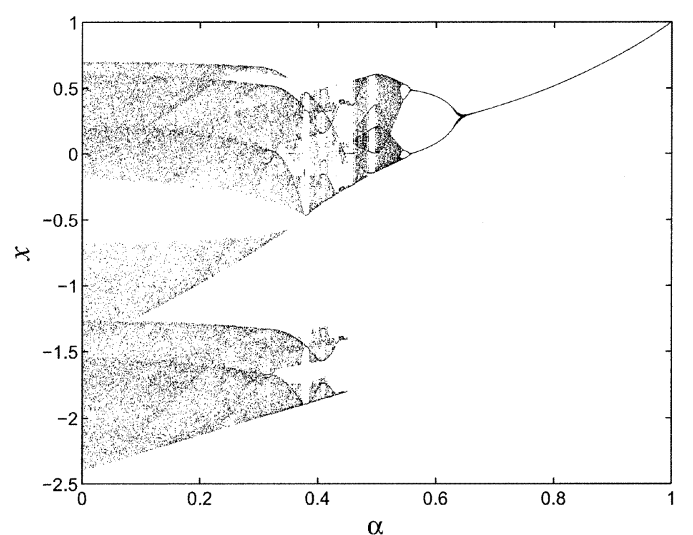

(a)

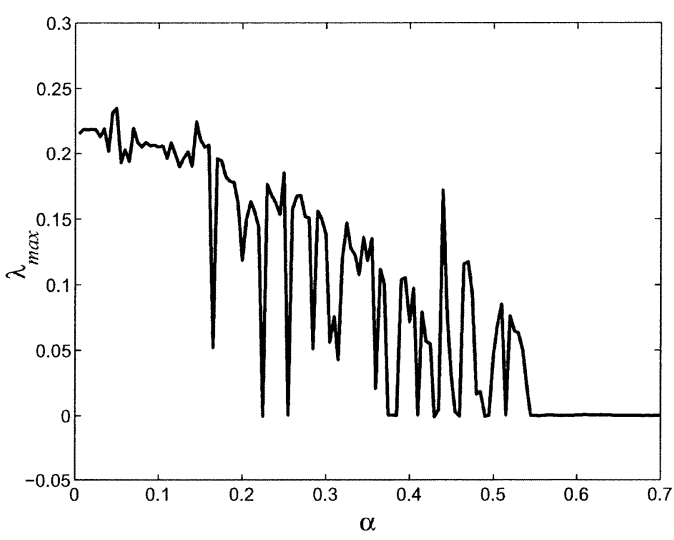

(c)

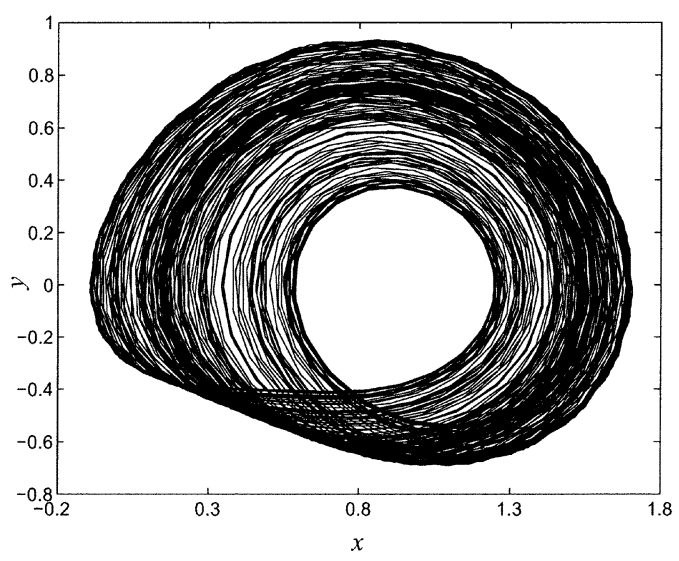

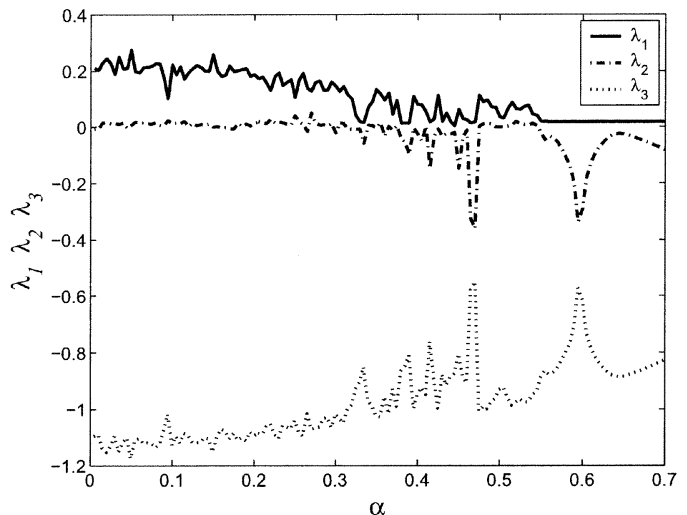

(b)

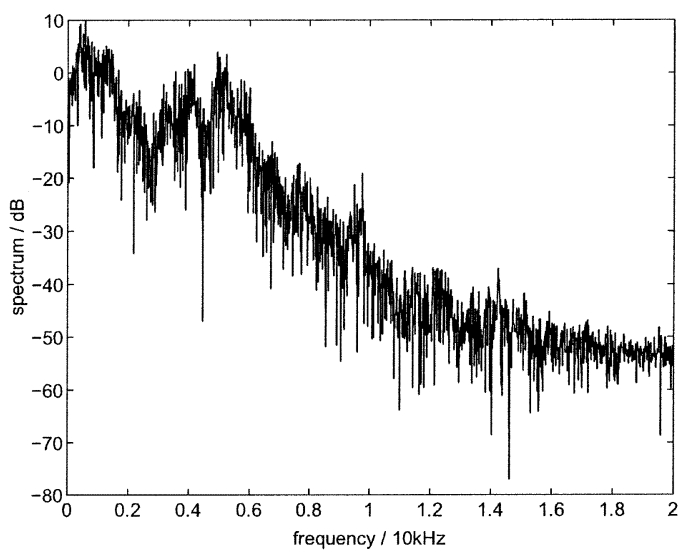

(d)

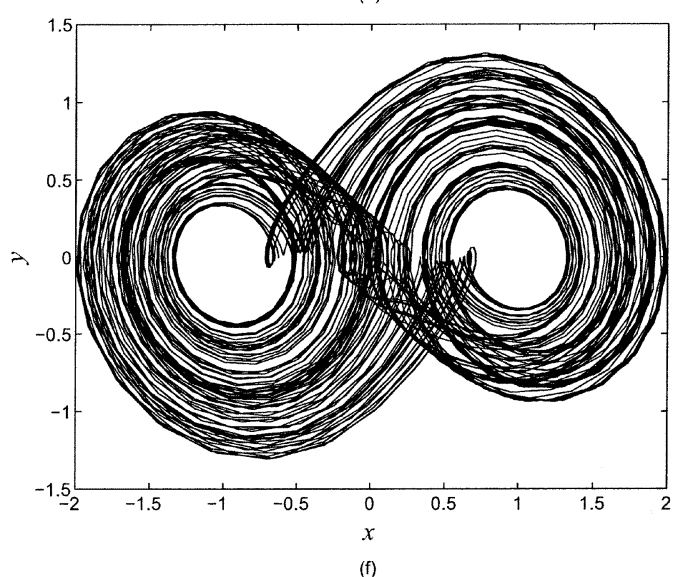

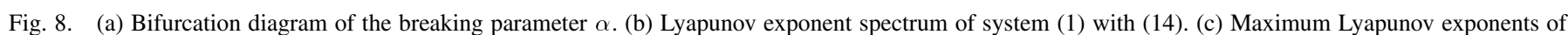
system (1) with (14). (d) Power spectrum of the double-scroll attractor. (e) Single-scroll attractor $(\alpha=0.52)$. (f) Double-scroll attractor $(\alpha=0.3)$.

scrolls of $f(x)$ have the same swings and widths; otherwise, all scrolls of $f(x)$ have different swings and widths. The detailed formulas are similar to those of the sawtooth wave, so they are omitted here.

3) Parameters $\alpha_{n}$ can also adjust the shapes and even the phase portrait of the multiscroll attractor. Assume that parameters $A, B$ are constants. Thus, the phase portraits will be away from the equilibrium points as the parameter $\alpha_{n}$ increases; and the phase portrait will be close to the equilibrium points as the parameter $\alpha_{n}$ decreases. Fig. 9(a) shows the case of the phase portrait being away from the equilibrium points, where $A=0.8, B=1.2, \alpha_{i}=$ $0.18(i=0, \pm 1, \pm 2$ ). Fig. 9(b) shows the case of the phase portrait being close to the equilibrium points, where $A=0.8, B=1.2, \alpha_{i}=0.018(i=0, \pm 1, \pm 2)$.

In the following, it is to further investigate the dynamical behaviors of system (1) with the triangular wave (15).

Denote the corresponding zeros of the segments with positive and negative slopes of the triangular wave $f_{1}(x)$ as $E_{q, n}^{+}$and $E_{q, n}^{-}$, respectively; that is

$$
\begin{aligned}
& E_{q, n}^{+}=\frac{2 n A}{B} \quad(n=0, \pm 1, \ldots, \pm M) \\
& E_{q, n}^{-}=\left(2 n-\frac{|n|}{n}\right) \frac{A}{B} \quad(n= \pm 1, \pm 2, \ldots, \pm M) .
\end{aligned}
$$



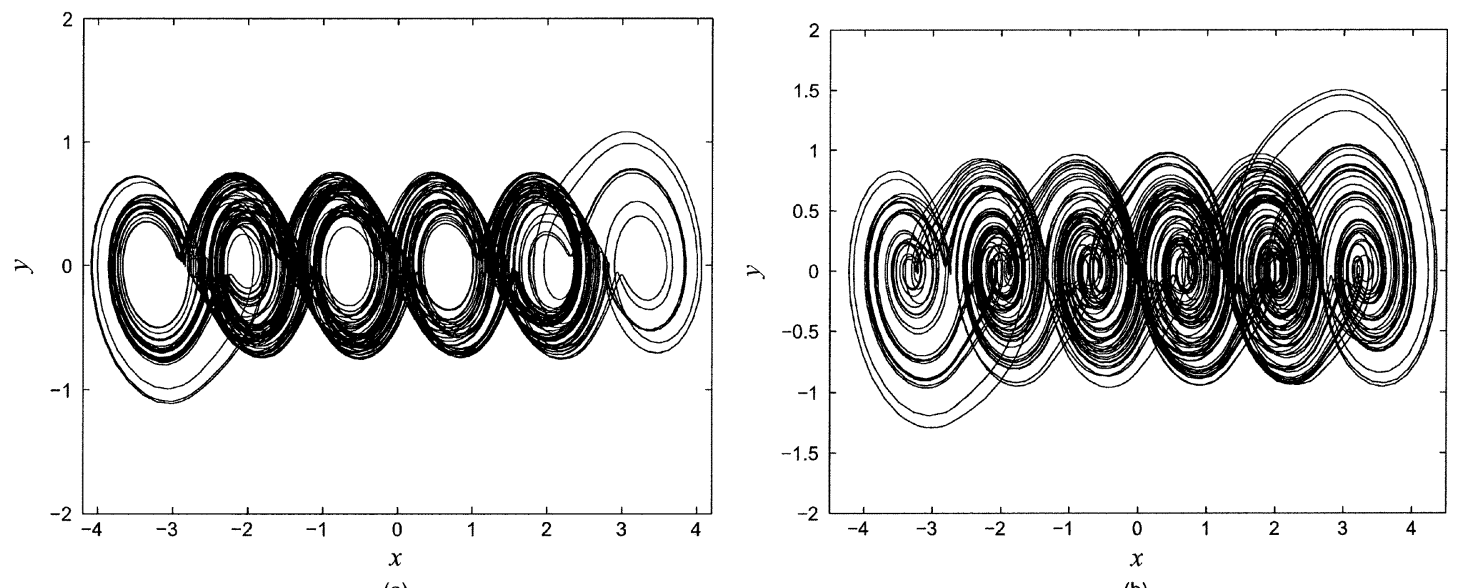

(a)

(b)

Fig. 9. 6-scroll chaotic attractor. (a) Phase portrait being away from equilibrium points $(\alpha=0.18)$. (b) Phase portrait being close to equilibrium points ( $\alpha=$ $0.018)$.

Let $\dot{x}=y$ and $\ddot{x}=z$. Then, the corresponding Jacobian matrices and their characteristic equations of the zeros $E_{q, n}^{+}$and $E_{q, n}^{-}$are

$$
J=\left(\begin{array}{ccc}
0 & 1 & 0 \\
0 & 0 & 1 \\
k & -\gamma & -\beta
\end{array}\right)
$$

and

$$
\lambda^{3}+\beta \lambda^{2}+\gamma \lambda-k=0 .
$$

where $k=((A-\alpha B) / \alpha)$ for the zeros $E_{q, n}^{+}$and $k=-B$ for the zeros $E_{q, n}^{-}$. Furthermore, the stabilities of equilibrium points $\left(E_{q, n}^{ \pm}, 0,0\right)$ of the jerk system (1) with (15) (or (16)) can be confirmed by using Lemma 1, (4), and (5) together.

Theoretical analysis shows that all equilibrium points $\left(E_{q, n}^{ \pm}, 0,0\right)$ can be classified into two different kinds: saddle points of index $1\left(E_{q, n}^{+}, 0,0\right)$ and saddle points of index 2 $\left(E_{q, n}^{-}, 0,0\right)$. That is, there exist saddle points of index 1 and saddle points of index 2 in the triangular wave. However, there only exists a saddle points of index 2 in the sawtooth wave. Therefore, the inherent mechanisms of chaos generation are different for the triangular wave and sawtooth wave.

For example, when $A=0.8, B=1.2, \alpha_{i}=0.18(i=$ $0, \pm 1, \pm 2$ ), system (1) has a 6 -scroll attractor as shown in Fig. 9(a). The corresponding eigenvalues are $\lambda_{1}^{+}=$ $1.1146, \lambda_{2,3}^{+}=-0.8573 \pm 1.4751 j$ for all $E_{q, n}^{+}$and $\lambda_{1}^{-}=-0.9237, \lambda_{2,3}^{-}=0.1619 \pm 1.1282 j$ for all $E_{q, n}^{-}$. Therefore, all $\left(E_{q, n}^{+}, 0,0\right)$ are saddle points of index 1 and all $\left(E_{q, n}^{-}, 0,0\right)$ are saddle points of index 2.

It follows from the above theoretical analysis that the eigenvalues $\lambda_{1}^{-}, \lambda_{2,3}^{-}$can drive the trajectories in the neighboring regions of $\left(E_{q, n}^{-}, 0,0\right)$ to rotate around the saddle points of index $2\left(E_{q, n}^{-}, 0,0\right)$ so as to form a scroll; the eigenvalues $\lambda_{1}^{+}, \lambda_{2,3}^{+}$can drive the trajectories in the neighboring regions of $\left(E_{q, n}^{+}, 0,0\right)$ to move away from the saddle points of index $1 E_{q, n}^{+}$and to go from a scroll to its neighboring scrolls so as to form the whole multiscroll chaotic attractor. Therefore, these saddle points are important to the formation of the multiscroll attractors.

\section{Designing Multiscroll Chaotic Attractors via Adjustable Transconductor Wave}

In this subsection, a smooth nonlinear function (the adjustable transconductor wave) is constructed to replace the sawtooth wave and triangular wave, to create multiscroll chaotic attractors in the jerk system (1).

To generate the chaotic attractor with an even number of scrolls, the adjustable transconductor wave is constructed as

$$
f_{1}(x)=\sum_{n=-M}^{M} A \tanh \left[C_{n}\left(x-\frac{2 n A}{B}\right)\right]-B x
$$

where $A, B, C_{n}$ are adjustable parameters, and $M \in N$.

Similarly, to create a chaotic attractor with an odd number of scrolls, the adjustable transconductor wave is constructed as

$$
f_{2}(x)=\sum_{\substack{n=-M \\ n \neq 0}}^{M} A \tanh \left[C_{n}\left(x-\left(2 n-\frac{|n|}{n}\right) \frac{A}{B}\right)\right]-B x
$$

where $A, B, C_{n}$ are adjustable parameters, and $M \in N$.

Notice that the parameters $A, B, C_{n}$ have the same physical meanings as the parameters $A, B, \alpha_{n}$ of the triangular wave.

Similarly, one can determine the stabilities of the equilibrium points. Fig. 10(a) and (b) shows the numerical simulation results of the 8- and 7-scroll attractors, respectively, where $A=2, B=$ $1, C_{n}=5$.

\section{Circuit IMPLEMENTATION FOR MUlTiscroll CHAOTIC ATTRACTORS}

In this section, some fundamental principles are discussed, for designing circuits to generate multiscroll chaotic attractors, especially $n$-scroll attractors with a large number of scrolls $(n>$ 10). Some experimental observations are also presented.

\section{A. Fundamental Principle for Circuit Design for Multiscroll Attractor}

Based on the operational principles of sawtooth wave and triangular wave, according to (1), (8), (9), (15), and (16), one can 


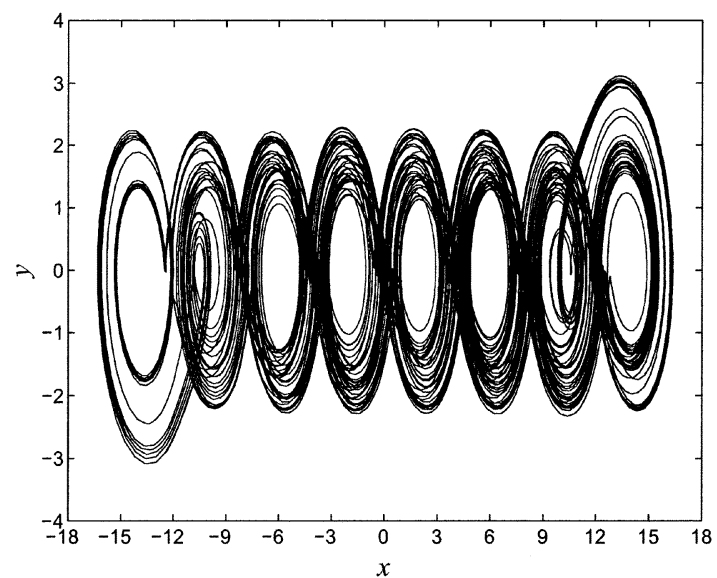

(a)

Fig. 10. Parameters $A=2, B=1, C_{n}=5$. (a) 7-scroll. (b) 8-scroll.

design a circuit diagram to realize various multiscroll chaotic attractors.

Fig. 11 shows the circuit diagram. This circuit diagram includes five different parts; that is, Part I: integrator $N_{0}$; Part II: sawtooth wave and triangular wave generator $N_{1}$; Part III: buffer $\mathrm{N}_{2}$; Part IV: switch linkages, including $K, K_{10}, K_{11}, K_{12}, K_{13}, K_{14}, K_{15}$; Part V: voltage-current conversion resistors $R 10 \sim R 15$.

Note that the buffer $N_{2}$ can greatly improve the load-ability of OP1, which is very important for generating more than 10 scrolls attractor by using a physical circuit. The number of scrolls can be completely controlled by the switchings of the switch linkages, $K_{10}, K_{11}, K_{12}, K_{13}, K_{14}, K_{15}$. The circuit diagram can physically realize $3-12$-scroll chaotic attractors by adjusting the switch linkages. Also, the generator $N_{1}$ can create sawtooth wave and triangular wave via the switching of the switch linkage $K$. The difference between the sawtooth wave oscillator and the triangular wave oscillator is the changing time between the charging and the discharging of the capacitor. When the output of the operational amplifier is a positive voltage, it is being charged rapidly to a small resistance value. When the output of the operational amplifier is a negative voltage, it is being charged gradually to a large resistance value. Here, $\left(1 /\left(R_{0} C_{0}\right)\right)$ is the integral constant of the integrator $N_{0}$, and the transformation factor of the time scale.

Assume that $R_{0}=1 \mathrm{k} \Omega$. Then, the transformation factor of the time scale will vary with $C_{0}$, which leads to the change of the spectrum range of the chaotic signal. In all experiments, $R_{0}=$ $1 \mathrm{k} \Omega$ and $C_{0}=33 \mathrm{nF}$. Moreover, all original devices shown in Fig. 11 are operational amplifiers of type TL082 with voltage supply $\pm 15 \mathrm{~V}$. The experimental results show that the saturated voltage of the operational amplifier is close to $\pm 13.5 \mathrm{~V}$. For convenience, all resistors shown in Fig. 11 are adjustable resistors with high precision, or potentiometers.

The circuit experimental results show that the dynamic ranges with high precision of the operational amplifiers are limited. Under the condition of small input signals, the input and output of the operational amplifier can keep a high operational precision. However, under the condition of large input signals, the

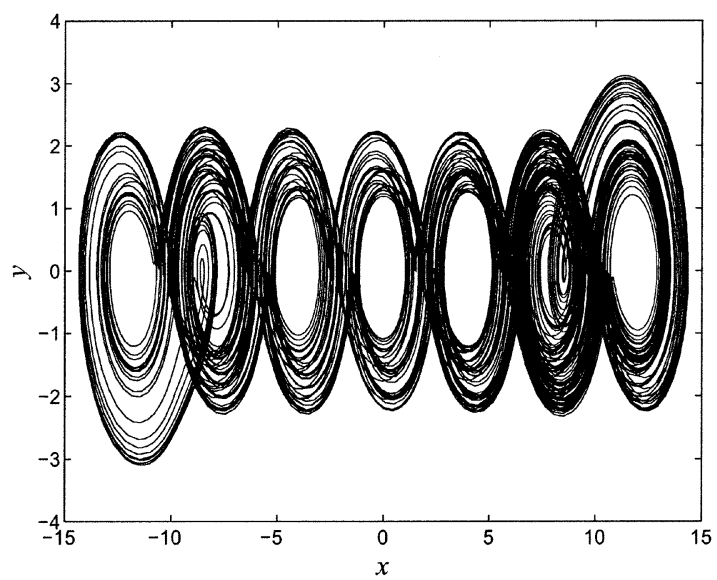

(b)

input and output of the operational amplifier cannot keep a high operational precision, which often causes an increasing error. Moreover, the parameter scatters of various operational amplifiers and other devices (such as resistors and capacitances) also increase the error. For these reasons, it is almost impossible for the physical chaotic circuit to generate chaotic attractors with a large number of scrolls, especially for more than ten scrolls in the attractor. Up to now, there does not seem to be any result reported in the literature on physical circuit implementation for chaotic attractors with more than ten scrolls.

To overcome these difficulties, one has to decrease the errors of the operational amplifiers as quickly as possible. Note that it is important to select suitable system parameters $A_{i}(i=$ $0, \ldots, M)$ and $B$ for the circuit implementation if one wants to generate attractors with more than ten scrolls. Here, we decrease the input signals of all operational amplifiers by decreasing the system parameters $A_{i}(i=0, \ldots, M)$. However, the values of $A_{i}(i=0, \ldots, M)$ cannot be too small for technical reasons. After numerous real circuit experimental trials, we select a set of suitable system parameters for $A_{i}(i=0, \ldots, 5)$ and $B$ as listed in Table I. According to (12), one can deduce the voltages $S_{i}(i=1, \ldots, 5)$ of the breakpoints for sawtooth wave and triangular wave, given in Tables IV and VII, respectively.

To generate four kinds of chaotic attractors with an even number of scrolls, including Types I, II, III, and IV, one needs to adjust all potentiometers $R_{4}$ in the sawtooth wave generator $N_{1}$ to ensure all input voltages of the operational amplifiers to satisfy the values listed in Table IV.

According to the circuit diagram shown in Fig. 11, when the switch linkage $K$ is off, one can get the relationship between the output voltages $x_{j}(j=0, \pm 1, \ldots, \pm 5)$ and input voltage $x$ for the operational amplifier; which is

$$
x_{j}=-\left|V_{\mathrm{sat}}\right| \operatorname{sgn}\left(x-S_{j}\right)
$$

where $j=0, \pm 1, \ldots, \pm 5, V_{\text {sat }} \approx 13.5 \mathrm{~V}$. Since the sawtooth wave is an odd function, $S_{-j}=-S_{j}$, whose values are listed in Table IV, where all voltages are in volt. 


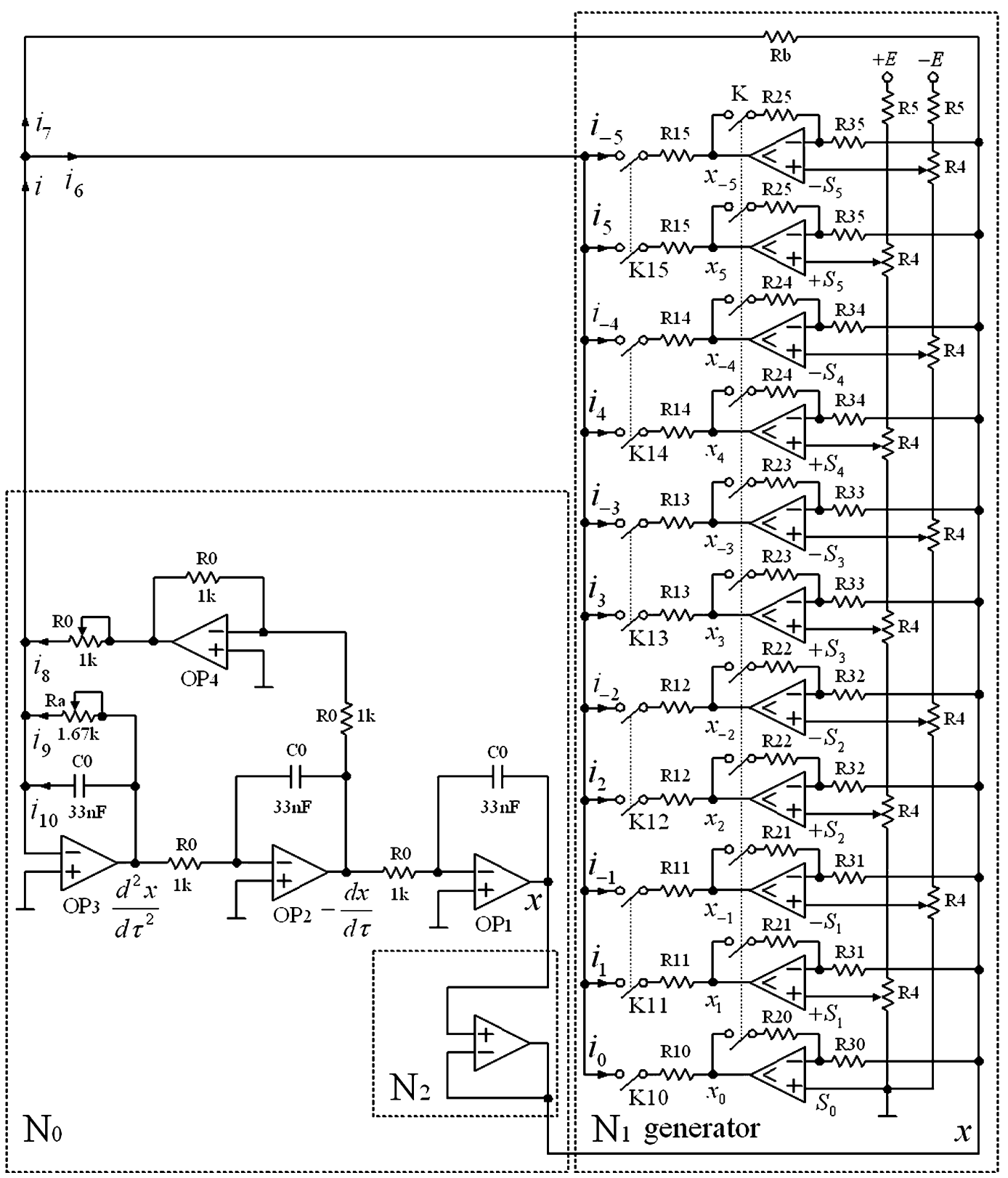

Fig. 11. Circuit diagram for realizing $n$-scroll attractors.

Denote the voltage-current conversion resistor of the output of the operational amplifier in $N_{1}$ as $R_{1 j}(j=0, \pm 1, \ldots, \pm 5)$. From the circuit diagram shown in Fig. 11, one has

$$
i_{j}=\frac{\left|V_{\mathrm{sat}}\right|}{R_{1 j}} \operatorname{sgn}\left(x-S_{j}\right)
$$

where $j=0, \pm 1, \ldots, \pm 5, R_{1,-j}=R_{1, j}$, and the units of the current $i_{j}(j=0, \pm 1, \ldots, \pm 5)$ are milliampere. Therefore

$$
i_{7}=-\frac{x}{R_{b}} \text {. }
$$

Define

$$
K_{1 j}= \begin{cases}1, & \text { if } K_{1 j} \text { is switched on } \\ 0, & \text { if } K_{1 j} \text { is switched off }\end{cases}
$$

where $j=0,1, \ldots, 5$. According to Fig. 11 and (22)-(23), one can get the total current of the sawtooth wave generator with an even number of scrolls, as follows:

$$
\begin{aligned}
i= & i_{6}+i_{7} \\
= & K_{10} i_{0}+K_{11}\left(i_{1}+i_{-1}\right)+K_{12}\left(i_{2}+i_{-2}\right) \\
& +K_{13}\left(i_{3}+i_{-3}\right)+K_{14}\left(i_{4}+i_{-4}\right)+K_{15}\left(i_{5}+i_{-5}\right)+i_{7} \\
= & \sum_{j=-M}^{M} \frac{\left|V_{\mathrm{sat}}\right|}{R_{1 j}} \operatorname{sgn}\left(x-S_{j}\right)-\frac{x}{R_{b}}
\end{aligned}
$$

where $M=1, \ldots, 5, R_{1,-j}=R_{1, j}$ for $j=0,1, \ldots, 5$, and the units of all currents are milliampere. 
TABLE IX

ON-OFF OF SWITCH LINKAGES $K_{10} \sim K_{15}$ AND NUMBER OF EVEN SCROLLS

\begin{tabular}{c|c|c|c|c|c|c}
\hline$K_{10}$ & $K_{11}$ & $K_{12}$ & $K_{13}$ & $K_{14}$ & $K_{15}$ & $2 M+2$ \\
\hline on & on & off & off & off & off & 4 \\
\hline on & on & on & off & off & off & 6 \\
\hline on & on & on & on & off & off & 8 \\
\hline on & on & on & on & on & off & 10 \\
\hline on & on & on & on & on & on & 12 \\
\hline
\end{tabular}

It follows from (8) and (25) that

$$
\left\{\begin{array}{l}
R_{10}=\frac{\left|V_{\text {sat }}\right|}{A_{0}} \\
R_{1 j}=\frac{2\left|V_{\text {sat }}\right|}{A_{j-1}+A_{j}} \\
R_{b}=\frac{1}{B}
\end{array}\right.
$$

where $j=1, \ldots, 5$, and the parameters $A_{j}(j=0, \ldots, 5)$ and $B$ are listed in Table I.

To generate an even number of $n$-scroll $(n=4,6,8,10,12)$ attractor from the circuit diagram shown in Fig. 11, let $K_{10}=$ $K_{11}=1$, and adjust the switch linkages $K_{12}, K_{13}, K_{14}, K_{15}$ based on Table IX.

Similarly, from the circuit diagram shown in Fig. 11, one can get the total current of the sawtooth wave generator with an odd number of scrolls, as follows:

$$
\begin{aligned}
i= & i_{6}+i_{7} \\
= & K_{11}\left(i_{1}+i_{-1}\right)+K_{12}\left(i_{2}+i_{-2}\right)+K_{13}\left(i_{3}+i_{-3}\right) \\
& +K_{14}\left(i_{4}+i_{-4}\right)+K_{15}\left(i_{5}+i_{-5}\right)+i_{7} \\
= & \sum_{\substack{j=-M \\
j \neq 0}}^{M} \frac{\left|V_{\text {sat }}\right|}{R_{1 j}} \operatorname{sgn}\left(x-S_{j}\right)-\frac{x}{R_{b}}
\end{aligned}
$$

where $M=1, \ldots, 5, R_{1,-j}=R_{1, j}$ for $j=0,1, \ldots, 5$, and the units of all currents are milliampere. Here, $R_{1 j}$ for $j=$ $0,1, \ldots, 5$ and $R_{b}$ are determined by (26), $S_{-j}=-S_{j}$ are given in Table VII, and $K_{1 j}$ for $j=0,1, \ldots, 5$ are defined in (24).

To create an odd number of $n$-scroll $(n=3,5,7,9,11)$ attractor from the circuit diagram shown in Fig. 11, let $K_{10}=$ $0, K_{11}=1$, and adjust the switch linkages $K_{12}, K_{13}, K_{14}, K_{15}$ based on Table X.

To generate four different kinds of multiscroll chaotic attractors with an odd number of scrolls, including Types I, II, III, and IV, one needs to adjust all potentiometers $R_{4}$ of the sawtooth wave generator $N_{1}$ to ensure all input voltages of operational amplifiers to satisfy the values listed in Table VII.

According to Table I and (26), one can calculate the voltagecurrent conversion resistors $R_{1 j}$ for $j=0,1, \ldots, 5$ and $R_{b}$, which are listed in Table XI.

\section{B. Example}

In this subsection, the operational principle of the circuit diagram shown in Fig. 11 is further explained via a simple example of triangular wave.

As can be seen from the circuit diagram shown in Fig. 11, when the switch linkages $K, K_{10}$ are switched on and the
TABLE X

ON-OFF OF SWITCH LINKAGES $K_{10} \sim K_{15}$ AND NUMBER OF ODD SCROLLS

\begin{tabular}{c|c|c|c|c|c|c}
\hline$K_{10}$ & $K_{11}$ & $K_{12}$ & $K_{13}$ & $K_{14}$ & $K_{15}$ & $2 M+1$ \\
\hline off & on & off & off & off & off & 3 \\
\hline off & on & on & off & off & off & 5 \\
\hline off & on & on & on & off & off & 7 \\
\hline off & on & on & on & on & off & 9 \\
\hline off & on & on & on & on & on & 11 \\
\hline
\end{tabular}

TABLE XI

RESISTOR VALUES OF $R_{1 j}(0 \leq j \leq 5)$ AND $R_{b}$ FOR FOUR DifFERENT TYPES OF ATTRACTORS

\begin{tabular}{c|c|c|c|c}
\hline Resistor & Type I & Type II & Type III & Type IV \\
\hline$R_{10}$ & $45 k \Omega$ & $27 k \Omega$ & $38.57 k \Omega$ & $54 k \Omega$ \\
\hline$R_{11}$ & $42 k \Omega$ & $28.13 k \Omega$ & $45 k \Omega$ & $54 k \Omega$ \\
\hline$R_{12}$ & $37.5 k \Omega$ & $30.7 k \Omega$ & $45 k \Omega$ & $54 k \Omega$ \\
\hline$R_{13}$ & $33.75 k \Omega$ & $33.75 k \Omega$ & $45 k \Omega$ & $54 k \Omega$ \\
\hline$R_{14}$ & $30.7 k \Omega$ & $37.5 k \Omega$ & $45 k \Omega$ & $54 k \Omega$ \\
\hline$R_{15}$ & $28.13 k \Omega$ & $42 k \Omega$ & $45 k \Omega$ & $54 k \Omega$ \\
\hline$R_{b}$ & $1 k \Omega$ & $1 k \Omega$ & $1 k \Omega$ & $1 k \Omega$ \\
\hline
\end{tabular}

other switch linkages $K_{1 j}(1 \leq j \leq 5)$ are switched off, the circuit diagram generates a double-scroll attractor. Obviously, when the absolute value of the input signal $x$ is less than the voltage value of the breakpoint $\alpha_{0}=\left(R_{30} / R_{20}\right)\left|V_{\text {sat }}\right|$; that is, $|x| \leq\left(R_{30} / R_{20}\right)\left|V_{\text {sat }}\right|$, the output voltage $x_{0}$ and the input voltage $x$ of the last sub-circuit in $N_{1}$ satisfy the linear relationship $x_{0}=-\left(\left(R_{30} x\right) / R_{20}\right)$. When the input signal $x$ exceeds the voltage value of the breakpoint, satisfying $|x|>\left(R_{30} / R_{20}\right)\left|V_{\text {sat }}\right|$, the operational amplifier enters into the saturated state and the output voltage is a constant, $V_{\text {sat }}= \pm 13.5 \mathrm{~V}$. That is

$$
x_{0}= \begin{cases}\left|V_{\text {sat }}\right|, & x<-\frac{R_{30}}{R_{20}}\left|V_{\text {sat }}\right| \\ -\frac{R_{20}}{R_{30}} x, & -\frac{R_{30}}{R_{20}}\left|V_{\text {sat }}\right| \leq x \leq \frac{R_{30}}{R_{20}}\left|V_{\text {sat }}\right| \\ -\left|V_{\text {sat }}\right|, & x>\frac{R_{30}}{R_{20}}\left|V_{\text {sat }}\right|\end{cases}
$$

where the units of the resistors are $\mathrm{k} \Omega$, and the unit of $x_{0}$ is volt.

According to the characteristic properties of the ideal operational amplifier and the reference direction of the current $i_{0}$, one has

$$
i_{0}=-\frac{x_{0}}{R_{10}}
$$

where the unit of the resistor is $\mathrm{k} \Omega$, and the unit of current $i_{0}$ is milliampere. Fig. 12(a) shows the voltage-ampere relationship for circuit $i_{0}$.

From the reference direction of the current $i_{7}$, one has

$$
i_{7}=-\frac{x}{R_{b}}
$$

where the unit of the resistor is $\mathrm{k} \Omega$, and the unit of current $i_{0}$ is milliampere. Fig. 12(b) shows the voltage-ampere relationship for current $i_{7}$.

According to the circuit diagram shown in Fig. 11, the entire current of $N_{1}$ is $i=i_{6}+i_{7}=i_{0}+i_{7}$. Fig. 12(c) shows the voltage-ampere relationship for the entire current $i$. Fig. 12(d) shows the function of $f(x)=R_{0} i$, where $R_{0}=1 \mathrm{k} \Omega$. Note that $f(x)$ and $i$ have the same diagrams but different units; that is, the unit of $i$ is milliampere and the unit of $f(x)$ is volt. 


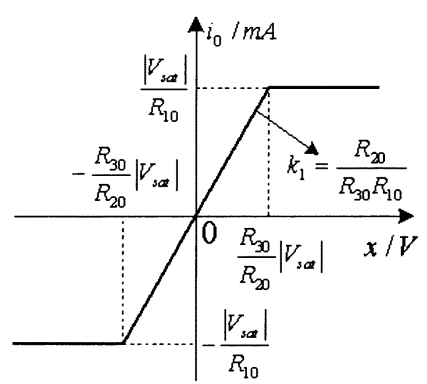

(a)

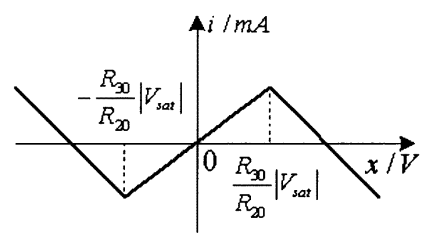

(c)

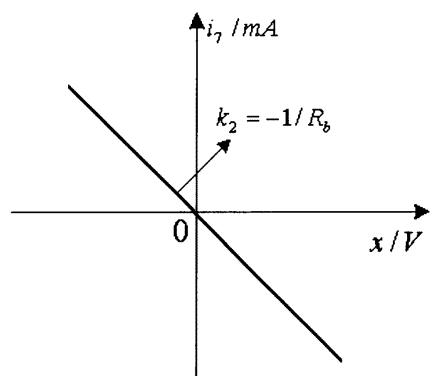

(b)

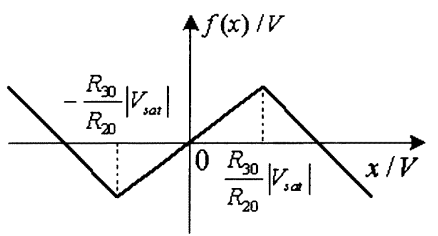

(d)
Fig. 12. Forming process for the triangular wave $f(x)$ in generator $N_{1}$. (a) Voltage-ampere relationship for current $i_{0}$. (b) Voltage-ampere relationship for current $i_{7}$. (c) Voltage-ampere relationship for synthesis current $i=i_{0}+i_{7}$. (d) Function relationship of $f(x)$.

Fig. 12 clearly reveals the forming process for the triangular wave $f(x)$ in generator $N_{1}$. The whole forming process can be divided into four different phases: Phase I [Fig. 12(a)]: the saturating process of current $i_{0}$; Phase II [Fig. 12(b)]: the forming process of current $i_{7}$; Phase III [Fig. 12(c)]: the synthesizing process of $i_{0}$ and $i_{7}$; Phase IV [Fig. 12(d)]: the forming process of $f(x)$.

Moreover, if the resistor $R_{20}$ is off, that is, the switch linkage $K$ of Fig. 11, is off, $R_{20} \rightarrow \infty$ and the voltage value of the breakpoint $\alpha_{0}=\left(R_{30} / R_{20}\right)\left|V_{\text {sat }}\right| \rightarrow 0$. Therefore, the triangular wave tends to the sawtooth wave in the limit case.

\section{Theoretical Analysis on the Multiscroll Chaotic Circuit}

In this subsection, the jerk circuit (1) is derived from the circuit diagram shown in Fig. 11.

According to circuit theory and the circuit diagram shown in Fig. 11, one can get the current equation

$$
i_{8}+i_{9}+i_{10}=i
$$

where $i$ is defined by (25) or (27). From the circuit diagram, one has

$$
\left\{\begin{array}{l}
i_{8}=\frac{1}{R_{0}} \frac{d x}{d \tau} \\
i_{9}=\frac{1}{R_{a}} \frac{d^{2} x}{d \tau^{2}} \\
i_{10}=C_{0} \frac{d}{d t}\left(\frac{d^{2} x}{d \tau^{2}}\right)
\end{array}\right.
$$

where $R_{0}=1 \mathrm{k} \Omega$. Multiplying both sides of (28) by $R_{0}$, from (28) and (29), one can deduce the following equation:

$$
R_{0} C_{0} \frac{d}{d t}\left(\frac{d^{2} x}{d \tau^{2}}\right)=-\frac{R_{0}}{R_{a}} \frac{d^{2} x}{d \tau^{2}}-\frac{d x}{d \tau}+f(x)
$$

where $R_{0}=1 \mathrm{k} \Omega, R_{a}=1.67 \mathrm{k} \Omega, \beta=\left(R_{0} / R_{b}\right)=0.6$, and $f(x) \in\left\{f_{1}(x), f_{2}(x)\right\}$, in which $f_{1}(x)$ and $f_{2}(x)$ are defined by (8) and (9) [or (15) and (16)], respectively.

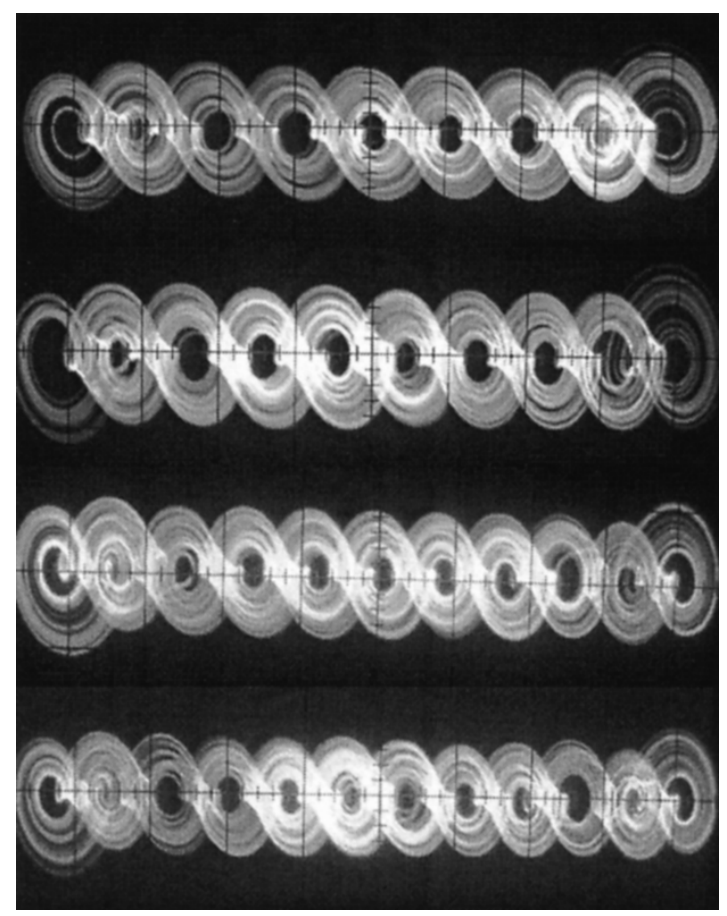

Fig. 13. Experimental observations of Type-IV $n$-scroll attractors. From up to down: (a) 9-scroll, where $x=0.5 \mathrm{~V} / \mathrm{div}, y=0.33 \mathrm{~V} / \mathrm{div}$; (b) 10 -scroll, where $x=0.55 \mathrm{~V} / \mathrm{div}, y=0.33 \mathrm{~V} / \mathrm{div}$; (c) 11 -scroll, where $x=0.6 \mathrm{~V} / \mathrm{div}, y=0.33$ V/div; (d) 12-scroll, where $x=0.66 \mathrm{~V} / \mathrm{div}, y=0.33 \mathrm{~V} / \mathrm{div}$.

It is noticed that the mathematical form of (28) does not change after multiplying both sides of (28) by $R_{0}$. However, the unit of (28) has been changed from milliampere to volt. Moreover, $\left(1 /\left(R_{0} C_{0}\right)\right)$ is the integrator constant of the integrator $N_{0}$ shown in Fig. 11, and it is also the transformation factor of the time scale. It is clear that (30) can be transformed into the jerk circuit (1) via a simple transformation $t=R_{0} C_{0} \tau$.

\section{Circuit Implementation}

In this subsection, the multiscroll chaotic attractors are experimentally confirmed via circuit design and oscilloscope observations.

Based on the circuit diagram shown in Fig. 11 and the calculated resistor values listed in Table XI, we have performed the following real physical experiments.

1) Switch off the switch linkage $K$, let $N_{1}$ be sawtooth wave, adjust the resistors $R_{1 j}$ for $j=0,1, \ldots, 5$ and $R_{b}$ from the type IV in Table XI, and change the status of the switch linkages $K_{1 j}$ for $j=0,1, \ldots, 5$. Then, we get the Type-IV sawtooth wave with 3-12 scrolls. Fig. 13 shows the oscilloscope-observed results for 9-12-scroll attractors.

2) Similarly, adjust the resistors $R_{1 j}$ for $j=0,1, \ldots, 5$ and $R_{b}$ from the Types I, II, III in Table XI. Then, one obtains three different kinds of sawtooth waves, including types I, II, III. Fig. 14 shows the oscilloscope-observed results for 11- and 12-scroll attractors.

3) Switch on the switch linkage $K$, and let $N_{1}$ be triangular wave. Then, we get various multiscroll attractors based on this triangular wave. Let $R_{30}=R_{31}=R_{32}=R_{33}=$ $R_{34}=R_{35}=1 \mathrm{k} \Omega$, and adjust the resistors values 


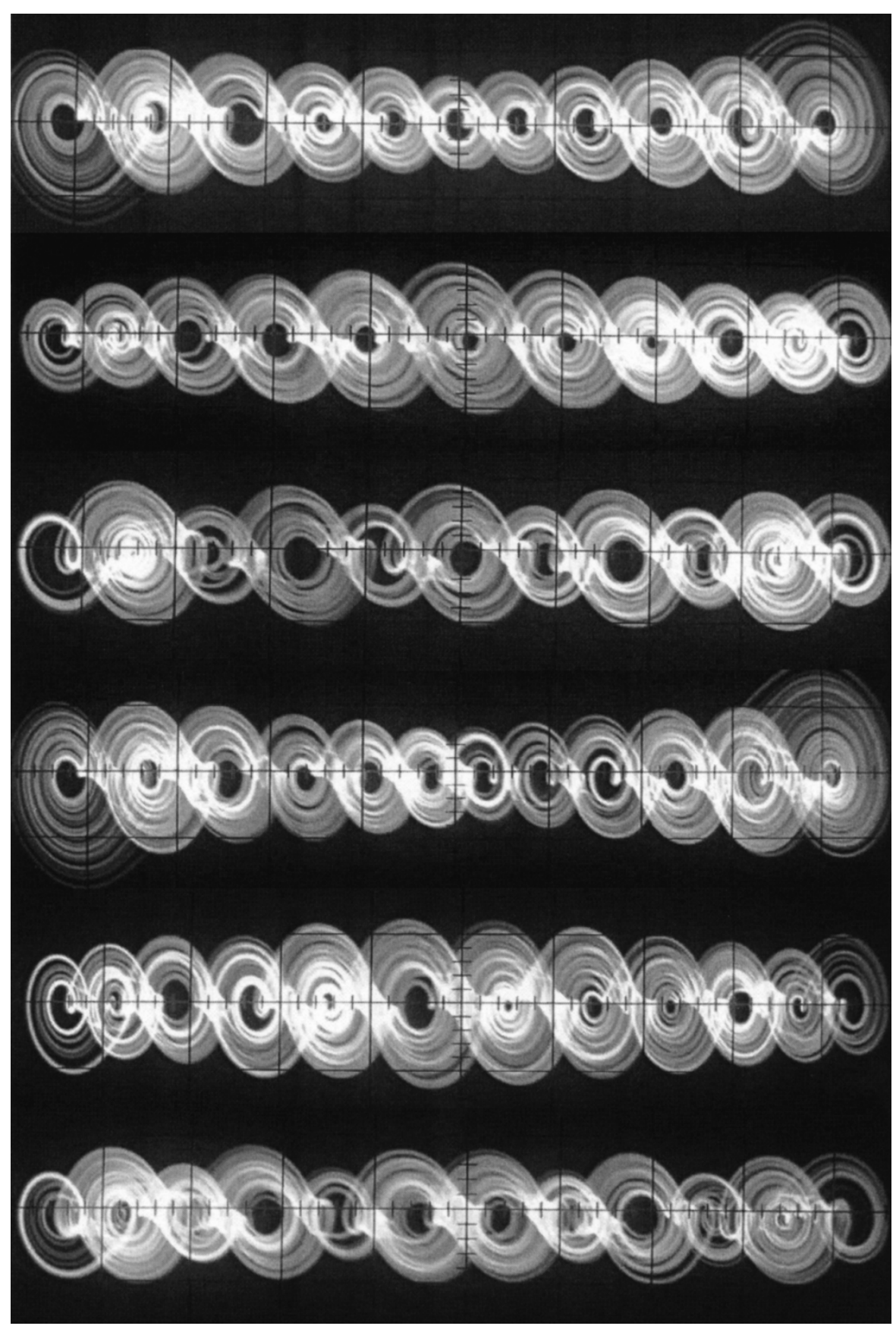

Fig. 14. Experimental observations of $n$-scroll attractors. From up to down: (a) 11 -scroll of Type I, $x=1.0 \mathrm{~V} / \mathrm{div}$, $y=0.4 \mathrm{~V} / \mathrm{div}$; (b) 11 -scroll of Type II, $x=0.95 \mathrm{~V} / \mathrm{div}, y=0.6 \mathrm{~V} / \mathrm{div}$; (c) 11 -scroll of Type III, $x=0.73 \mathrm{~V} / \mathrm{div}, y=0.4 \mathrm{~V} / \mathrm{div}$; (d) 12 -scroll of Type I, $x=1.1 \mathrm{~V} / \mathrm{div}, y=0.4 \mathrm{~V} / \mathrm{div}$; (e) 12 -scroll of Type II, $x=1.05 \mathrm{~V} / \mathrm{div}, y=0.5 \mathrm{~V} / \mathrm{div}$; (f) 12 -scroll of Type III, $x=0.8 \mathrm{~V} / \mathrm{div}, y=0.4 \mathrm{~V} / \mathrm{div}$.

$R_{2 j}$ for $j=0,1, \ldots, 5$. Then, one can change the corresponding values of the breakpoints $\alpha_{j}$ for $j=0,1, \ldots, 5$ of the triangular wave. The function relationships between $\alpha_{j}$ and $R_{2 j}, R_{3 j}$ are

$$
\alpha_{j}=\frac{R_{3 j}}{R_{2 j}}\left|V_{\text {sat }}\right|
$$

where $j=0,1, \ldots, 5$. Notice that $\alpha_{j}$ for $j=0,1, \ldots, 5$ can modify the shapes and even the phase portraits of the triangular waves. The phase portraits move away from the equilibrium points as $\alpha_{j}$ increases; and they will be close to the equilibrium points as $\alpha_{j}$ decreases. Fig. 15 shows the oscilloscope-observed results for 5-scroll and 6-scroll attractors.

\section{CONCLUSION}

This paper has developed a nonlinear modulating function approach for creating $n$-scroll chaotic attractors from a general jerk circuit. The dynamic mechanism and chaos generation condition of the general jerk circuit have been investigated by analyzing the system stability. A novel block circuit diagram 


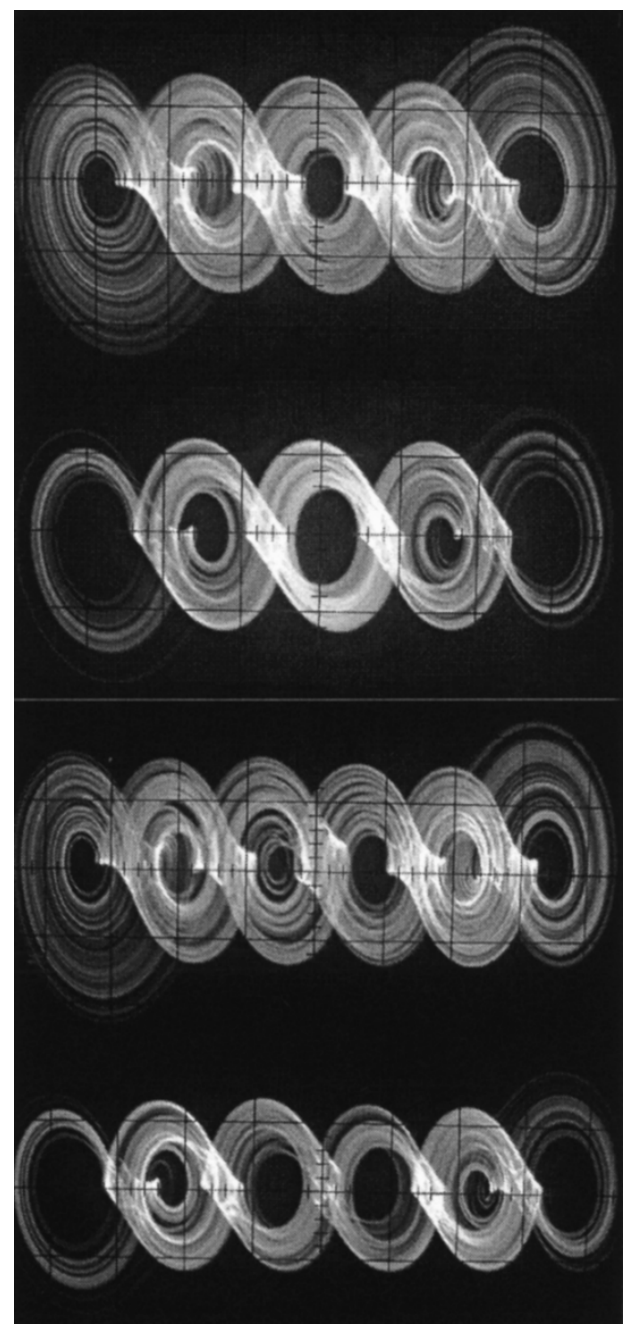

Fig. 15. Experimental observations of $n$-scroll attractors. From up to down: (a) 5-scroll attractor with phase portraits close to equilibrium points, where $x=0.8 \mathrm{~V} / \mathrm{div}, y=0.8 \mathrm{~V} / \mathrm{div}$, and $\alpha=0.018$; (b) 5-scroll attractor with phase portraits away from equilibrium points, where $x=0.8 \mathrm{~V} / \mathrm{div}, y=$ $0.8 \mathrm{~V} / \mathrm{div}$, and $\alpha=0.18$; (c) 6 -scroll attractor with phase portraits close to equilibrium points, where $x=0.9 \mathrm{~V} / \mathrm{div}, y=0.8 \mathrm{~V} / \mathrm{div}$, and $\alpha=0.018$; (d) 6-scroll attractor with phase portraits away from equilibrium points, where $x=0.9 \mathrm{~V} / \mathrm{div}, y=0.8 \mathrm{~V} / \mathrm{div}$, and $\alpha=0.18$.

has been designed for hardware implementation of various 3-12-scroll chaotic attractors; it includes integrator, sawtooth wave and triangular wave generators, buffer, switch linkages, and voltage-current conversion resistors. Moreover, the recursive formulas of system parameters and real physical circuit parameters have been rigorously derived, useful for hardware implementation of a chaotic attractor with a large number of scrolls.

The novel circuit design approach developed in this paper has many advantages over the classical methods: (i) one can arbitrarily design the swings, widths, slopes, breakpoints, equilibrium points, shapes, and even the phase portraits of the multiscroll chaotic attractors by using the adjustable sawtooth wave and triangular wave functions; (ii) all system design parameters and real physical circuit parameters can be rigorously derived from the recursive formulas (10)-(13). Therefore, our circuit design method has high reliability, diversity, and practicability. More importantly, the adjustability of the sawtooth wave and triangular wave as well as the rigorous recursive formulas provide a theoretical principle for physically realizing chaotic attractors with a large number of scrolls.

Our physical experiment results have verified that the high precision region of the operational amplifiers is limited. That is, the input and output of the operational amplifier have a high operational precision for small input signals. However, it is very difficult for the input and output of the operational amplifier to retain the high operational precision under the condition of large input signals. Also, there are other technical reasons that cause difficulties in hardware implementation for generating chaotic attractors with a large number of scrolls, such as the parameters scatters, the dynamic range of the available physical devices, and the variations of the input and output impedances of the real operational amplifier. This reveals the reason why there are very few (if any) results reported in the literature for physical circuit implementation of chaotic attractors with more than ten scrolls. Nevertheless, in this paper we have already overcome these difficulties by accurately calculating the parameters and designing a suitable block circuit diagram, thereby physically realizing a 12-scroll chaotic attractor.

It should be pointed out that one can arbitrarily design the desired swings, widths, slopes, breakpoints, equilibrium points, shapes, and even the phase portraits of the $n$-scroll chaotic attractor by using the proposed systematic methodology. In particular, the adjustability of nonlinear modulating function and the rigorous recursive formulas together form a theoretical basis for hardware implementation of various chaotic attractors with a large number of scrolls. Furthermore, the block circuit structure, adjustability, diversity, and high-reliability of the circuitry design will further facilitate some engineering applications of multiscroll chaotic attractors, such as monolithic IC realization via the CMOS technology.

\section{REFERENCES}

[1] G. Chen and X. Yu, Chaos Control: Theory and Applications. Berlin, Germany: Springer-Verlag, 2003.

[2] J. A. K. Suykens and J. Vandewalle, "Quasilinear approach to nonlinear systems and the design of $n$-double scroll $(n=1,2,3,4, \ldots)$," Proc. Inst. Elect. Eng., pt. G, vol. 138, no. 5, pp. 595-603, Oct. 1991.

[3] — " "Generation of $n$-double scrolls $(n=1,2,3,4, \ldots)$," IEEE Trans. Circuits Syst. I, Fundam. Theory Appl., vol. 40, no. 11, pp. 861-867, Nov. 1993.

[4] J. A. K. Suykens, A. Huang, and L. O. Chua, "A family of $n$-scroll attractors from a generalized Chua's circuit," AEU-Int. J. Electron. Commun., vol. 51, no. 3, pp. 131-138, May 1997.

[5] J. A. K. Suykens and L. O. Chua, " $n$-double scroll hypercubes in 1-D CNNs," Int. J. Bifurc. Chaos, vol. 7, no. 8, pp. 1873-1885, Aug. 1997.

[6] P. Arena, S. Baglio, L. Fortuna, and G. Manganaro, "Generation of $n$-double scrolls via cellular neural networks," Int. J. Circuit Theor. Appl., vol. 24, no. 3, pp. 241-252, May/Jun. 1996.

[7] M. A. Aziz-Alaoui, "Differential equations with multispiral attractors," Int. J. Bifurc. Chaos, vol. 9, no. 6, pp. 1009-1039, Jun. 1999.

[8] M. E. Yalcin, J. A. K. Suykens, and J. Vandewalle, "Hyperchaotic $n$-scroll attractors," in Proc. IEEE Workshop on Nonlinear Dynamics of Electronic Systems (NDES 2000), Catania, Italy, May 2000, pp. 25-28.

[9] M. E. Yalcin, S. Ozoguz, J. A. K. Suykens, and J. Vandewalle, " $n$-scroll chaos generators: A simple circuit model," Electron. Lett., vol. 37, no. 3, pp. 147-148, Feb. 2001.

[10] T. Matsumoto, L. O. Chua, and M. Komuro, "The double scroll," IEEE Trans. Circuits Syst., vol. 32, no. 8, pp. 798-817, Aug. 1985.

[11] L. O. Chua, M. Komuro, and T. Matsumoto, "The double scroll family," IEEE Trans. Circuits Syst., vol. 33, no. 11, pp. 1072-1118, Nov. 1986.

[12] S. Nakagawa and T. Saito, "An RC OTA hysteresis chaos generator," IEEE Trans. Circuits Syst. I, Fundam. Theory Appl., vol. 43, no. 12, pp. 1019-1021, Dec. 1996. 
[13] A. S. Elwakil and M. P. Kennedy, "Chaotic oscillators derived from Saito's double-screw hysteresis oscillator," IEICE Trans. Fund., vol. E82-A, no. 9, pp. 1769-1775, Sep. 1999.

[14] K. S. Tang, G. Q. Zhong, G. Chen, and K. F. Man, "Generation of $n$-scroll attractors via sine function," IEEE Trans. Circuits Syst. I, Fundam. Theory Appl., vol. 48, no. 11, pp. 1369-1372, Nov. 2001.

[15] G. Zhong, K. F. Man, and G. Chen, "A systematic approach to generating $n$-scroll attractors," Int. J. Bifurc. Chaos, vol. 12, no. 12, pp. 2907-2915, Dec. 2002.

[16] A. S. Elwakil and M. P. Kennedy, "Construction of classes of circuitindependent chaotic oscillators using passive-only nonlinear devices," IEEE Trans. Circuits Syst. I, Fundam. Theory Appl., vol. 48, no. 3, pp. 289-307, Mar. 2001

[17] S. Ozoguz, A. S. Elwakil, and K. N. Salama, " $n$-scroll chaos generator using nonlinear transconductor," Electron. Lett., vol. 38, no. 14, pp. 685-686, Jul. 2002.

[18] J. Lü, T. Zhou, G. Chen, and X. Yang, "Generating chaos with a switching piecewise-linear controller," Chaos, vol. 12, no. 2, pp. 344-349, Jun. 2002.

[19] J. Lü, X. Yu, and G. Chen, "Generating chaotic attractors with multiple merged basins of attraction: A switching piecewise-linear control approach," IEEE Trans. Circuits Syst. I, Fundam. Theory Appl., vol. 50, no. 2, pp. 198-207, Feb. 2003.

[20] M. E. Yalcin, J. A. K. Suykens, J. Vandewalle, and S. Ozoguz, "Families of scroll grid attractors," Int. J. Bifurc. Chaos, vol. 12, no. 1, pp. 23-41, Jan. 2002.

[21] D. Cafagna and G. Grassi, "Hyperchaotic coupled Chua circuits: An approach for generating new $n \times m$-scroll attractors," Int. J. Bifurc. Chaos, vol. 13, no. 9, pp. 2537-2550, Sep. 2003.

[22] — " "New 3D-scroll attractors in hyperchaotic Chua's circuit forming a ring," Int. J. Bifurc. Chaos, vol. 13, no. 10, pp. 2889-2903, Oct. 2003.

[23] J. Lü, X. Yu, and G. Chen, "Switching control for multiscroll chaos generation: An overview," Proc. Phys. Control, vol. 2, pp. 420-428, Aug. 20-22, 2003.

[24] J. Lü, "Switching control: From simple rules to complex chaotic systems," J. Syst. Sci. Compl., vol. 16, no. 3, pp. 404-413, Jul. 2003.

[25] F. Han, X. Yu, Y. Wang, Y. Feng, and G. Chen, " $n$-scroll chaotic oscillators by second-order systems and double-hysteresis blocks," Electron. Lett., vol. 39, no. 23, pp. 1636-1638, Nov. 2003.

[26] F. Han, J. Lü, X. Yu, G. Chen, and Y. Feng, "Generating multiscroll chaotic attractors via a linear second-order hysteresis system," Dyn.Contin. Discr. Impuls. Syst. Ser. B, vol. 12, no. 1, pp. 95-110, 2005.

[27] J. Lü, F. Han, X. Yu, and G. Chen, "Generating 3-D multiscroll chaotic attractors: A hysteresis series switching method," Automatica, vol. 40, no. 10, pp. 1677-1687, Oct. 2004.

[28] J. Lü, G. Chen, X. Yu, and H. Leung, "Design and analysis of multiscroll chaotic attractors from saturated function series," IEEE Trans. Circuits Syst. I, Reg. Papers, vol. 51, no. 12, pp. 2476-2490, Dec. 2004.

[29] , "Generating multiscroll chaotic attractors via switching control," in Proc. 5th Asian Control Conf., Melbourne, Australia, Jul. 20-23, 2004, pp. 1763-1771.

[30] S. M. Yu, Z. G. Ma, S. S. Qiu, S. G. Peng, and Q. H. Lin, "Generation and synchronization of $n$-scroll chaotic and hyperchaotic attractors in fourth-order systems," Chin. Phys., vol. 13, no. 3, pp. 317-328, Mar. 2004.

[31] T. Matsumoto, L. O. Chua, and K. Kobayashi, "Hyperchaos: Laboratory experiment and numerical confirmation," IEEE Trans. Circuits Syst., vol. 33, no. 11, pp. 1143-1147, Nov. 1986.

[32] M. E. Yalcin, J. A. K. Suykens, and J. Vandewalle, "On the realization of $n$-scroll attractors," in Proc. IEEE Int. Symp. Circuits and Systems (ISCAS'99), vol. 5, May-Jun. 1999, pp. 483-486.

[33] - "Experimental confirmation of 3- and 5-scroll attractors from a generalized Chua's circuit," IEEE Trans. Circuits Syst. I, Fundam. Theory Appl., Fundam. Theory Appl., vol. 47, no. 3, pp. 425-429, Mar. 2000.

[34] A. S. Elwakil and M. P. Kennedy, "Systematic realization of a class of hysteresis chaotic oscillators," Int. J. Circuit Theor. Appl., vol. 28, no. 4, pp. 319-334, Jul./Aug. 2000.

[35] A. S. Elwakil, K. N. Salama, and M. P. Kennedy, "A system for chaos generation and its implementation in monolithic form," in Proc. IEEE Symp. Circuits Syst. (ISCAS'00), Geneva, Switzerland, May 28-31, 2000, pp. 217-220.

[36] S. M. Yu, S. S. Qiu, and Q. H. Lin, "New results of study on generating multiple-scroll chaotic attractors," Sci. China Ser. F, vol. 46, no. 2, pp. 104-115, Feb. 2003.

[37] J. Lü, G. Chen, and Y. Yu, "Asymptotic analysis of a modified Lorenz system," Chin. Phys. Lett., vol. 19, no. 9, pp. 1260-1263, Sep. 2002.
[38] G. Chen and J. Lü, Dynamics of the Lorenz System Family: Analysis, Control, and Synchronization. Beijing, China: Science Press, 2003.

[39] S. J. Linz and J. C. Sprott, "Elementary chaotic flow," Phys. Lett. A, vol 259, no. 3-4, pp. 240-245, Aug. 1999.

[40] J. C. Sprott, "Some simple chaotic flows," Phys. Rev. E, vol. 50, no. 2, pp. R647-R650, Aug. 1994.

[41] _ "Simple chaotic systems and circuits," Amer. J. Phys., vol. 68, no 8, pp. 758-763, Aug. 2000.

[42] _ "A new class of chaotic circuit," Phys. Lett. A, vol. 266, no. 1, pp. 19-23, Feb. 2000.

[43] M. Wada, Y. Nishio, and A. Ushida, "Chaotic itinerancy phenomena on coupled $n$-double scrolls chaotic circuits," in Proc. IEEE Int. Symp. Circuits and Systems (ISCAS'99), vol. 5, Orlando, FL, May-Jun. 1999, pp. 487-490.

[44] M. E. Yalcin, J. A. K. Suykens, and J. Vandewalle, "A double scroll based true random bit generator," in Proc. IEEE Int. Symp. Circuits and Systems (ISCAS'04), vol. 4, Vancouver, BC, Canada, May 23-26, 2004, pp. 581-584.

[45] P. Coullet, C. Tresser, and A. Arneodo, "Transition to stochasticity for a class of forced oscillators," Phys. Lett. A, vol. 72, no. 4-5, pp. 268-270, Jul. 1979.

[46] A. Arneodo, P. Coullet, and C. Tresser, "Possible new strange attractors with spiral structure," Commun. Math. Phys., vol. 79, no. 4, pp. 573-579, 1981.

[47] N. F. Rul'kov, A. R. Volkovskii, A. Rodriguez-Lozano, E. Del Rio, and M. G. Velarde, "Mutual synchronization of chaotic self-oscillators with dissipative coupling," Int. J. Bifurc. Chaos, vol. 3, pp. 669-676, Sep. 1992.

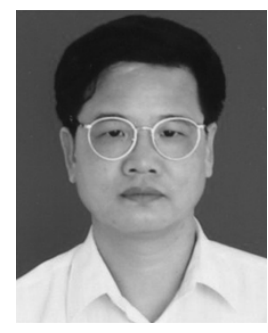

Simin Yu received the B.Sc. degree in electronics from Yunnan University, Kunming, China, in 1983, the M.E. degree in radio communication engineering, and the Ph.D. degree in nonlinear circuits and systems both from the South China University of Technology, Guangzhou, China, in 1996 and 2001, respectively.

Currently, he is a full Professor in the College of Automation, Guangdong University of Technology, Guangzhou, China. His research interests include the design and analysis of nonlinear circuits, computer simulations, and wireless communications. He has published more than 30 research journal papers in the fields of chaos in electronic circuits and chaotic communications.

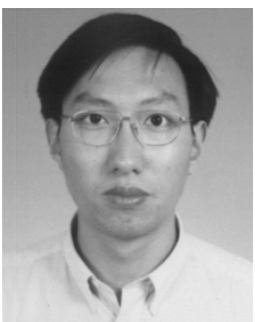

Jinhu Lü (M'03) received the B.Sc. degree in mathematics, the M.Sc. degree in applied mathematics, and $\mathrm{Ph} . \mathrm{D}$. degree in applied mathematics, from Hubei Normal University, Wuhan University, and Chinese Academy of Sciences, Beijing, China, in 1997, 2000, and 2002, respectively.

From January to April of 2002, he was a Research Assistant, and from August to November of 2003, a Senior Research Associate at the Centre for Chaos Control and Synchronization, City University of Hong Kong, Hong Kong SAR, China. From January to May 2003, he was a Visiting Research Fellow in the School of Electrical and Computer Engineering, Royal Melbourne Institute of Technology University, Melbourne, Australia. From June 2002 to February 2004, he was a Postdoctoral Research Fellow, and from March 2004, an Assistant Professor with the Institute of Systems Science, Academy of Mathematics and Systems Sciences, Chinese Academy of Sciences, Beijing, China. Currently, he is a Postdoctoral Fellow in the Department of Electrical and Computer Engineering, University of Calgary, Calgary, $\mathrm{AB}$, Canada. He is the author of two research monographs and more than 50 research journal papers published in the fields of control and synchronization of complex dynamical systems.

Dr. Lü is now serving as the member of the Technical Committee on Nonlinear Circuits and Systems of the IEEE Circuits and Systems Society. He received the Presidential Outstanding Research Award from the Chinese Academy of Sciences in 2002 and the 100 National Best Ph.D. Theses Award from the Office of Academic Degrees Committee of the State Council and the Ministry of Education of China in 2004. 


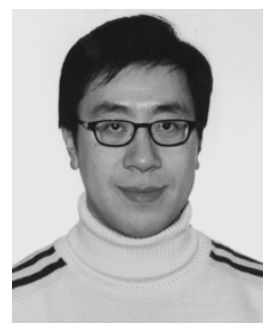

Henry Leung (M'90) received the Ph.D. degree in electrical and computer engineering from McMaster University, Hamilton, ON, Canada.

$\mathrm{He}$ is now a Professor in the Department of Electrical and Computer Engineering, University of Calgary, Calgary, AB, Canada. Before this, he was with the Defence Research Establishment, Ottawa, ON, Canada, where he was involved in the design of automated systems for air and maritime multisensor surveillance. His research interests include chaos, computational intelligence, data mining, nonlinear signal processing, multimedia, radar, sensor fusion and wireless communications.

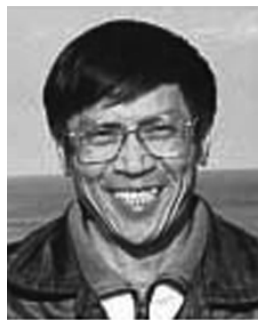

Guanrong Chen (M'87-SM'92-F'96) received the M.Sc. degree in computer science from the Sun Yat-sen (Zhongshan) University, Guangzhou, China and the Ph.D. degree in applied mathematics from Texas A\&M University, College Station.

Currently, he is a Chair Professor and the Founding Director of the Centre for Chaos Control and Synchronization at the City University of Hong Kong, Hong Kong. He has (co)authored 15 research monographs and advanced textbooks, more than $300 \mathrm{SCI}$ journal papers, and about 200 refereed conference papers, published since 1981 in the fields of nonlinear system dynamics and controls. Among his publications are the research monographs and edited books entitled Hopf Bifurcation Analysis: A Frequency Domain Approach (Singapore: World Scientific, 1996), From Chaos to Order: Methodologies, Perspectives and Applications (Singapore: World Scientific, 1998), Controlling Chaos and Bifurcations in Engineering Systems (Boca Raton, FL: CRC Press, 1999), Chaos in Circuits and Systems (Singapore: World Scientific, 2002), as well as Chaos Control and Bifurcation Control (New York: Springer, 2003).

Prof. Chen served and is serving as Chief Editor, Deputy Chief Editor, Advisory Editor, Features Editor, and Associate Editors for eight international journals including the IEEE TRANSACTIONS ON CIRCUITS AND SYSTEMS, IEEE TRANSACTIONS ON AUTOMATIC CONTROL, and The International Journal of Bifurcation and Chaos. He received the 1998 Harden-Simons Prize for the Outstanding Journal Paper Award from the American Society of Engineering Education, the 2001 M. Barry Carlton Best Annual Transactions Paper Award from the IEEE Aerospace and Electronic Systems Society, the 2002 Best Paper Award from the Institute of Information Theory and Automation, Academy of Science of the Czech Republic, and the 2005 Guillemin-Cauer Best Annual Transactions Paper Award from the IEEE Circuits and Systems Society. He is Honorary Professor of the Central Queensland University, Australia, as well as Honorary Guest-Chair Professor of more than ten Universities in China. He is a Fellow of the IEEE for his fundamental contributions to the theory and applications of chaos control and bifurcation analysis. 